\title{
Microtubule-dependent apical restriction of recycling endosomes sustains adherens junctions during morphogenesis of the Drosophila tracheal system
}

\author{
Pierre-Marie Le Droguen, Sandra Claret, Antoine Guichet* and Véronique Brodu*
}

\begin{abstract}
Epithelial remodelling is an essential mechanism for organogenesis, during which cells change shape and position while maintaining contact with each other. Adherens junctions (AJs) mediate stable intercellular cohesion but must be actively reorganised to allow morphogenesis. Vesicle trafficking and the microtubule (MT) cytoskeleton contribute to regulating AJs but their interrelationship remains elusive. We carried out a detailed analysis of the role of MTs in cell remodelling during formation of the tracheal system in the Drosophila embryo. Induction of MT depolymerisation specifically in tracheal cells shows that MTs are essential during a specific time frame of tracheal cell elongation while the branch extends. In the absence of MTs, one tracheal cell per branch overelongates, ultimately leading to branch break. Three-dimensional quantifications revealed that MTs are crucial to sustain E-Cadherin (Shotgun) and Par-3 (Bazooka) levels at AJs. Maintaining E-Cadherin/Par-3 levels at the apical domain requires de novo synthesis rather than internalisation and recycling from and to the apical plasma membrane. However, apical targeting of E-Cadherin and Par-3 requires functional recycling endosomes, suggesting an intermediate role for this compartment in targeting de novo synthesized E-Cadherin to the plasma membrane. We demonstrate that apical enrichment of recycling endosomes is dependent on the MT motor Dynein and essential for the function of this vesicular compartment. In addition, we establish that E-Cadherin dynamics and MT requirement differ in remodelling tracheal cells versus planar epithelial cells. Altogether, our results uncover an MT-Dynein-dependent apical restriction of recycling endosomes that controls adhesion by sustaining Par-3 and E-Cadherin levels at AJs during morphogenesis.
\end{abstract}

KEY WORDS: Microtubules, Adherens junctions, Recycling endosomes

\section{INTRODUCTION}

Epithelial remodelling is an essential mechanism in the threedimensional shaping of developing organisms (Bryant and Mostov, 2008), which relies on cell rearrangements while maintaining the cell-cell adhesion required to ensure tissue integrity.

Epithelial cells adhere to each other by adherens junctions (AJs), which contain the cell-adhesion molecule E-Cadherin (E-Cad) and its cytoplasmic binding partners $\beta$-catenin $(\beta$-cat) and $\alpha$-catenin; $\alpha$-catenin ensures the link with the cytoskeleton. The maintenance and remodelling of AJs require accurate regulation of E-Cad

Institut Jacques Monod, CNRS and University Paris Diderot, 15 Rue H. Brion, Paris 75205, Cedex 13, France.

*Authors for correspondence (guichet.antoine@ijm.univ-paris-diderot.fr; brodu.veronique@ijm.univ-paris-diderot.fr)

Received 3 June 2014; Accepted 18 November 2014 dynamics by polarity factors and through membrane trafficking (Wirtz-Peitz and Zallen, 2009; Shivas et al., 2010). Integration of cytoskeletal networks with AJs regulates cell-cell interactions and coordinates tissue morphogenesis. The relationship between AJs and acto-myosin has been extensively studied and reveals the fundamental role of acto-myosin and its regulators in the establishment, maintenance and remodelling of cell contacts (Cavey and Lecuit, 2009).

Several studies have proposed a functional interplay between cadherins and the microtubule (MT) cytoskeleton, either in cell culture or from in vivo models, but without providing to date a comprehensive picture (Brieher and Yap, 2013). In cell culture, E-Cad engaged in homophilic adhesion can exert instructive effects that influence MT organisation at the cell cortex (Chausovsky et al., 2000). In a reverse scenario, MTs are necessary to maintain junctional integrity. Depolymerisation of MTs disrupts the morphology and organisation of E-Cad-based contacts in monolayers of polarised thyroid cells (Yap et al., 1995; Waterman-Storer et al., 2000) and Caco2 cells (Meng et al., 2008). This role is assumed by plus ends of dynamic MTs, which stabilise E-Cad at cell-cell contacts (Stehbens et al., 2006) through regulation of the acto-myosin network (Ratheesh et al., 2012). MTs can also regulate AJs by means of vesicular trafficking. N-Cadherin accumulates at AJs through kinesin-based transport on MTs (Chen et al., 2003; Teng et al., 2005). However, MT requirement in this process remains unclear. In MDCK cells, MTs participate in a specific transport from the Golgi towards the AJs, but their depletion does not prevent E-Cad-mediated cell-cell contacts (Nejsum and Nelson, 2007).

In the Drosophila embryo, MTs play a central role in the assembly of AJs. At the onset of polarity establishment during cellularisation, Par-3 [Bazooka (Baz) - FlyBase] recruits the cadherin-catenin complex and promotes AJ assembly in an MT-Dynein-dependent process (Harris and Peifer, 2005). However, during gastrulation, Par-3 and E-Cad [Shotgun (Shg) - FlyBase] exhibit proper apical localisation in a Dynein mutant context (Harris and Peifer, 2005). Furthermore, the absence of MTs lowers E-Cad levels at the AJs of epidermal cells but without affecting overall cell polarity or epithelium integrity (Jankovics and Brunner, 2006; Bulgakova et al., 2013). Altogether, these data acquired in developing tissue suggest that MTs might be required during epithelial morphogenesis for the establishment of cell-cell contacts but not for their maintenance.

Using the same approach as Bulgakova et al. (2013) and Jankovics and Brunner (2006), we previously observed that the absence of MTs affects the integrity of the Drosophila embryonic respiratory organ, also referred to as the tracheal system (Brodu et al., 2010). This discrepancy prompted us to investigate the functional requirement of the MT network for AJ assembly and/or maintenance during epithelial morphogenesis of the 3D tracheal 
system. This tubular network arises from clusters of ectodermal cells on either side of the ten embryonic segments. By stage 11, the cells of each tracheal placode invaginate and thereafter migrate to form the different branches, which by stage 13 fuse with those of adjacent metameres (Uv et al., 2003). Importantly, once tracheal cells invaginate, the absence of further cell division prevents MT reorganisation during the cell cycle. Thus, the role of MTs during cell remodelling can only be accounted for by changes in cell shape and cell rearrangements. We showed previously that, during placode invagination, the MT nucleation centre moves from the centrosome towards the apical domain of tracheal cells, from where MTs are then nucleated (Brodu et al., 2010). This dramatic MT network reorganisation is crucial for tracheal branch formation.

Here we focus on an analysis of dorsal branch (DB) and dorsal trunk (DT) formation, the remodelling of which has been investigated in numerous studies (Ribeiro et al., 2004; Caussinus et al., 2008). At the onset of DB formation at stage 13, cells are aligned in a side-by-side arrangement, all AJs being intercellular and visible as two parallel lines (Fig. 1A). During stages 14 and 15, this alignment becomes destabilised by the pulling force generated by the dorsal movement of the leading cells toward the source of Bnl (FGF), forcing the cells to shift their relative position and to intercalate. As cells move dorsally, intercellular AJs of adjacent cells are successively replaced by autocellular AJs. Finally, elongated cells adopt an end-to-end arrangement, and AJs form one straight line of autocellular junctions and small rings of intercellular junctions around the luminal space (Ribeiro et al., 2004; Caussinus et al., 2008).

In this study, we uncover the molecular and cellular consequences of MT depletion during the collective migration of tracheal cells. MT absence leads to cell overstretching during branch elongation and ultimately to branch break. We quantify how MTs sustain a correct distribution of E-Cad and Par-3 at AJs of tracheal cells and demonstrate that E-Cad and Par-3 levels require the functional recycling endosome compartment, which operates as an intermediate between the de novo synthesis of E-Cad in the Golgi apparatus and its targeting to the AJs. In addition, we show that the MT motor Dynein is required for the apical enrichment of recycling endosomes and thus for the targeting of Par-3 and E-Cad toward AJs. Altogether, our data reveal an MT-dependent apical restriction of recycling endosomes that promotes adhesion by sustaining Par-3 and E-Cad levels at AJs during tracheal branch extension.

\section{RESULTS}

\section{MTs interact functionally with E-Cad to ensure DB extension}

The UAS/GAL4 system was used to specifically overexpress the MT-severing enzyme Spastin in tracheal cells only after placode invagination. From stage 12 onwards, $\alpha$-tubulin is not detected in Spastin-overexpressing placode compared with wild type (WT) (Fig. 1C,H). In addition, we examined two $\alpha$-tubulin post-
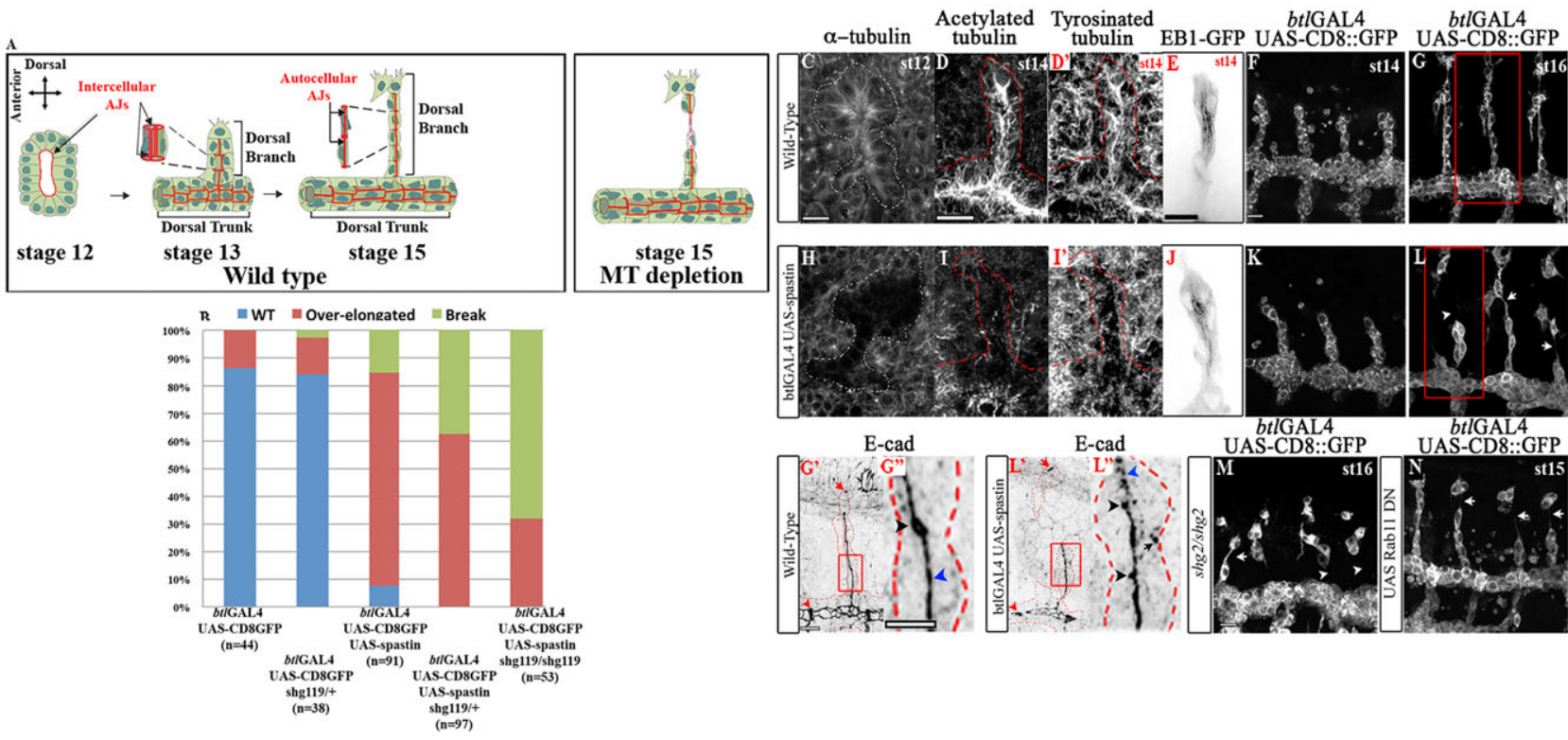

Fig. 1. MTs functionally interact with E-Cad for proper dorsal branch (DB) extension. (A) Schematic representation of cell intercalation during tracheal branch extension in WT and MT-depleted contexts. Green, cell bodies; blue, nuclei; red, AJs; grey, lumen. After placode invagination (stage 12, left), all tracheal cells are situated side-by-side and joined by intercellular AJs. As of stage 13 (middle), cells of the DB start to intercalate. At stage 15, intercalated cells show an autocellular, straight-line AJ (right). In the absence of MTs, cell overelongation is observed during branch elongation, which accompanies reduction of AJs. Here, and in the remaining figures, anterior is left and dorsal is up; red dashed lines highlight the tracheal tissue defined by the expression of the CD8::GFP chimeric protein. Expression of UAS transgenes is driven specifically in tracheal cells using the bt/-GAL4 driver and only DBs of central metameres A4 to A7 are considered. (B) The percentage of WT (blue), overelongated (red) or broken (green) DBs in different genetic contexts. (C,H) The MT network, as labelled with an anti- $\alpha$-tubulin antibody, is largely depleted in Spastin-overexpressing tracheal cells as of stage $12(\mathrm{H})$ as compared with WT (C). Stage 14 embryos are labelled for acetylated tubulin and tyrosinated tubulin in WT $\left(D, D^{\prime}\right)$ or Spastin-overexpressing tracheal cells $\left(I, I^{\prime}\right)$. In this context, signals are strongly reduced in tracheal cells as compared with surrounding tissues Time projection of UAS-Eb1-GFP expression at stage 14 reveals growing MTs in WT (E) or Spastin-overexpressing (J) tracheal cells. Track numbers corresponding to Eb1-GFP projections are extremely reduced in the Spastin-overexpression context. (F,G,K,L) Expression of the membrane-bound form CD8::GFP in WT (F,G) or Spastin-overexpressing (K,L) tracheal cells at stage $14(\mathrm{~F}, \mathrm{~K})$ and stage $16(\mathrm{G}, \mathrm{L})$. Cell overelongation (arrows) and branch breaks (arrowheads) become apparent as of stage 16. $\left(G^{\prime}, L^{\prime}\right)$ Enlarged views of boxed regions in $G$ and $L$, respectively, showing $E$-Cad distribution. MT depletion ( $\left.L^{\prime}\right)$ does not prevent the establishment of new cell-cell contacts between fusion cells of DBs (red arrows) and of dorsal trunk (DT) (red arrowheads). (G", $\left.L^{\prime \prime}\right)$ Enlarged views of $G^{\prime}$ and $L^{\prime}$, respectively. E-Cad distribution in the DB is less homogenous after MT depletion ( $\mathrm{L}^{\prime \prime}$ ) as compared with WT ( $\left.\mathrm{G}^{\prime \prime}\right)$. Gaps in the line formed by autocellular junctions are visible (compare blue arrowheads). Loops of intercellular junctions are smaller than in WT (black arrowheads). Intracellular accumulation of E-Cad is visible as dots (black arrow). (M,N) Cell overelongation in the DB (white arrows) is visible in $s g^{2}$ mutant tracheal cells (M) or when Rab11DN is overexpressed (N). Scale bars: $10 \mu \mathrm{m}$, except $5 \mu \mathrm{m}$ in $\mathrm{G}^{\prime \prime}$ 
Table 1. Analysis of the tracheal defects induced by MT depletion

A

\begin{tabular}{|c|c|c|c|c|}
\hline & Wild type & MT depleted & MT depleted over-elongated cells & MT-depleted other cells \\
\hline Stalk length of DB & $47.8 \pm 4(n=12)$ & $49.8 \pm 6.1(n=16)$ & nd & nd \\
\hline Tracheal cell length & $13.5 \pm 2.5(n=36)$ & nd & $24.5 \pm 4.3(n=16)$ & $10.3 \pm 1.8(n=41)$ \\
\hline
\end{tabular}

B

\begin{tabular}{lll}
\hline & DB base & DB tip \\
Overelongation position & $26.70 \%(n=4)$ & $73.30 \%(n=11)$ \\
Length of overelongated tracheal cell & $22.9 \pm 4.7$ & $25.1 \pm 4.2$
\end{tabular}

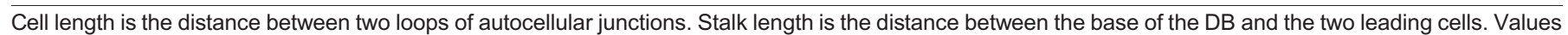

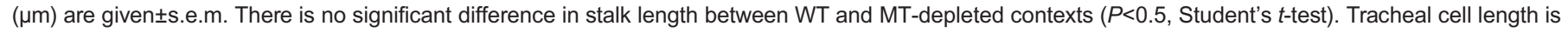

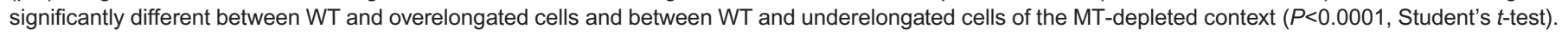
nd, not determined

translational modifications: tyrosination, which is associated with newly assembled MT fibres; and acetylation, which occurs on stable MTs (Janke and Bulinski, 2011). Both signals are strongly decreased, indicating that levels of dynamic and stable MT networks are substantially reduced in all of the forming branches (Fig. 1D, $\mathrm{D}^{\prime}, \mathrm{I}_{1} \mathrm{I}^{\prime}$ ), but with an emphasis on the DB. Accordingly, the number of Eb1 comets, corresponding to elongating MT fibres (Akhmanova and Steinmetz, 2008), is greatly reduced in the Spastin-overexpressing DB compared with the WT (Fig. 1E,J; supplementary material Fig. S1I). Altogether, Spastin overexpression in tracheal cells leads to an almost complete loss of the MT network during branch elongation, to leave just a few dynamic fibres.

Surprisingly, MT depletion does not lead to a collapse of the entire tracheal system and does not prevent overall DB migration, even though some defects in the direction of DB growth are occasionally observed $(12.5 \%, n=80)$. The stalk length between the base of the DB and the two leading cells is unchanged compared with WT (Table 1). In addition, no defects in initiation of the secretion of luminal products are detected (supplementary material Fig. S1A,B; data not shown). Lumen interruption is observed as a result of fusion defects between branches (supplementary material Fig. S1B) (Gervais et al., 2012).

The most striking phenotype observed in $77 \%$ of the cases (Fig. 1B) involves tracheal cells connected by long cytoplasmic threads (Fig. 1G,L, as schematised in Fig. 1A). This extreme cell elongation is visible either at the base or at the tip of the DB stalk (Table 1) and does not depend on the number of cells per DB (supplementary material Fig. S1J). In addition, this phenotype appears progressively during branch elongation. It is not yet visible at stage 14 (Fig. 1F,K) but becomes apparent from stage 15 onwards, when the response to FGF chemoattraction still exerts a pulling force on the DB. Using high-resolution live imaging, we confirmed that this phenotype, which we refer to as cell overelongation, is established progressively during branch elongation once intercalation is completed (supplementary material Movies 1 and 2). As a result, levels of the luminal product Piopio and levels of E-Cad-marked AJs are gradually lowered in the long cytoplasmic threads formed by the overelongated cells (supplementary material Fig. S1C,D). Tracheal cell overelongation ultimately leads to branch break in between two cells (15\% of cases) (Fig. 1B; supplementary material Movies 1 and 2). The overelongated cell measures $24.5 \pm 4.3 \mu \mathrm{m}$ $(n=16)$ as compared with $13.5 \pm 2.5 \mu \mathrm{m}(n=36)$ for WT cells (Table 1). This increase in cell length can occur at any position within the stalk (Table 1). As the stalk length remains comparable between WT and MT-depleted contexts, the overelongation of a single tracheal cell leads to the underelongation of its neighbours [10.3 $\pm 1.8 \mu \mathrm{m}(n=41)$ as compared with $13.5 \pm 2.5 \mu \mathrm{m}]$.

To investigate whether cell overelongation and branch break could be due to a reduction in adhesion between cells, DB formation was examined in various mutant contexts for E-Cad. In embryos homozygous for the amorphic allele $s h g^{2}$, defects similar to those observed in MT-depleted tracheal cells are detected (Fig. 1M). In addition, in embryos heterozygous for the hypomorphic allele shg ${ }^{119}, 2 \%$ branch break and $13 \%$ cell overelongation are observed (Fig. 1B). The phenotypes detected with such genetic combinations are more severe when MTs are depolymerised (supplementary material Fig. S1G,H), with $37 \%$ branch break and $63 \%$ cell overelongation (Fig. 1B). Furthermore, phenotypes become more extreme when examined in $s h g^{119}$ homozygous embryos, in which $68 \%$ branch break and $32 \%$ cell overelongation are visible (Fig. 1B). These results demonstrate that the MT network and AJs functionally interact to maintain DB integrity.

We then examined E-Cad distribution in an MT-depleted tracheal system. As revealed by a straight line of AJs, tracheal cells of the stage $16 \mathrm{DB}$ accomplish intercalation properly in the absence of MTs (Fig. $1 \mathrm{G}^{\prime}, \mathrm{L}^{\prime}$ ). In addition, tracheal cells maintain their capacity to establish new cellular junctions between metameres at the level of the DT or the DB (arrowheads in Fig. 1L'). However, the distribution of E-Cad at AJs is less homogeneous than in WT (Fig. 1G", $\mathrm{L}^{\prime \prime}$ ). The loop formed by intercellular junctions of two adjacent tracheal cells is smaller than in control embryos, suggesting that adhesion between tracheal cells is reduced compared with WT (Fig. $1 \mathrm{G}^{\prime \prime}, \mathrm{L}^{\prime \prime}$; supplementary material Fig. S1K). This could explain how tracheal cells, being less adhesive, gradually overelongate in response to the pulling force. Finally, we observed an abnormal intracellular distribution of E-Cad, visible as dots (arrows in Fig. 1L"). These results therefore indicate that the MT network is required to maintain the correct E-Cad distribution at AJs.

\section{MTs sustain E-Cad and Par-3 at AJs during DB extension}

We aimed to detect E-Cad from the beginning of DB formation to further understand how defaults in E-Cad distribution occur in the absence of MTs. At stage 14, an important intracellular accumulation of E-Cad, visible as large dots, is detected in all DB cells (Fig. $2 \mathrm{C}^{\prime}, \mathrm{F}^{\prime}$ ). To assess whether the presence of intracellular E-Cad dots depends on the intercalation state, DTs that are devoid of cell rearrangement were examined (Fig. 2F", $F^{\prime \prime} 2$ ). In addition, the Spalt transcription factor was overexpressed in the DB to inhibit cell intercalation (Shaye et al., 2008) (supplementary material Fig. S3A,B). In both cases, E-Cad dots are visible at stage 14, indicating that the intracellular accumulation of E-Cad does not depend on the intercalation state. 

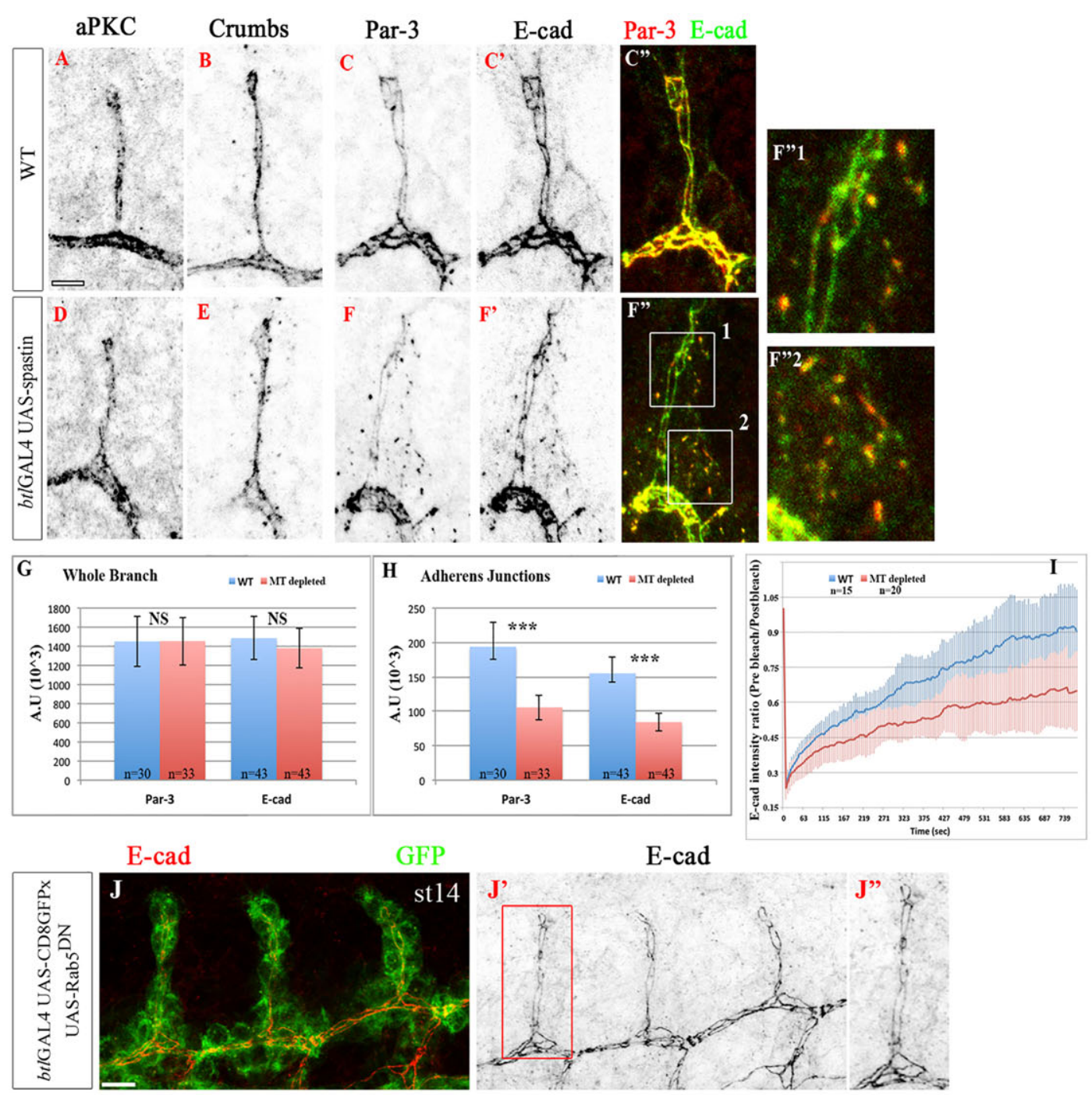

E-cad
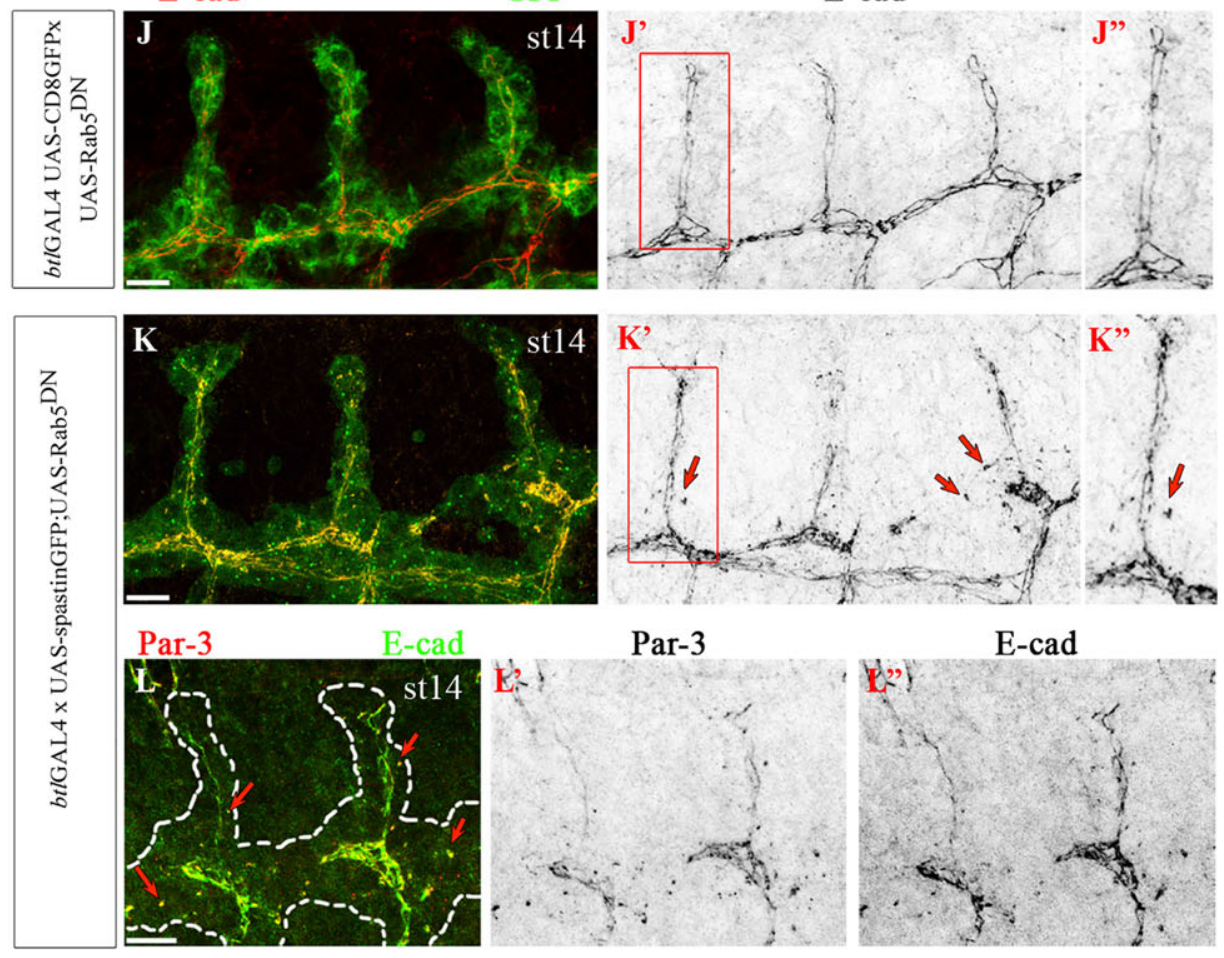

Par-3

E-cad
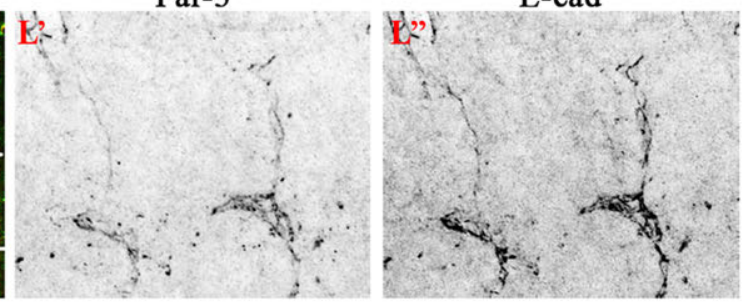

Fig. 2. MTs sustain E-Cad and Par-3 in AJs during DB extension. (A-F") DB of stage 14 embryos in WT (A-C") and MT-depleted (D-F") contexts. aPKC (A,D) and $\mathrm{Crb}(\mathrm{B}, \mathrm{E})$ distributions are unchanged in the absence of MTs. Par-3 $(\mathrm{C}, \mathrm{F})$ and E-Cad $\left(\mathrm{C}^{\prime}, \mathrm{F}^{\prime}\right)$ perfectly colocalise in WT $\left(\mathrm{C}^{\prime \prime}\right)$ and form large intracellular accumulations visible as dots in absence of MTs $\left(\mathrm{F}^{\prime \prime}\right)$. The boxed regions (1 and 2) in F" are enlarged on the right. (G,H) Quantifications of Par-3 and E-Cad immunofluorescence in WT and MT-depleted contexts, within the entire DB $(G)$ or restricted to AJs $(H)$. A.U, arbitrary fluorescence units. Error bars indicate s.e. m.; NS, not significant; *** $P<0.001$ (Student's $t$-test). (I) Timecourse of percentage fluorescence recovery relative to the pre-bleach value of E-Cad-GFP in WT or in MT-depleted contexts. Curves represent time point averages of independent experiments. Error bars indicate s.d. (J-J") Blocking E-Cad internalisation by overexpressing Rab5DN inhibits intercalation in the DB as visualised by parallel lines of E-Cad ( $\mathrm{J}^{\prime}$, magnified in $\left.\mathrm{J}^{\prime \prime}\right)$. (K-K") Overexpression of Rab5DN in MTdepleted cells induces intracellular accumulation of E-Cad (K', magnified in $\mathrm{K}^{\prime \prime}$; arrows). (L-L") These intracellular dots of E-Cad ( $\left.\mathrm{L}^{\prime \prime}\right)$ also contain Par-3 ( $\mathrm{L}^{\prime}$ ) (arrows in L). Scale bars: $5 \mu \mathrm{m}$ in A; $10 \mu \mathrm{m}$ in J-L. 
We then examined the distribution of apical polarity markers known to regulate E-Cad distribution towards the plasma membrane and at AJs in the epithelia. The apical distribution of aPKC (Fig. 2A,D) and Crumbs (Crb) (Fig. 2B,E) is not altered in the absence of MTs and no large intracellular dots are visible. In addition, the overall organisation of the actin network is unaffected in the MT-depleted context (supplementary material Fig. S2B,D), indicating that the intracellular accumulation of E-Cad relies only on the MT network alteration and is not an indirect consequence of a modification of the actin network. By contrast, Par-3 is detected as ectopic large dots, in contrast to WT, at stage 14 (Fig. 2C,F). The Par-3-positive dots colocalise with E-Cad (Fig. 2C",F"). Similar results are obtained for the distribution of $\beta$-cat (Armadillo FlyBase) (supplementary material Fig. S2A-A",C-C"), indicating that the intracellular dots correspond to the AJ complex.

To determine whether MT depletion leads to an intracellular accumulation of AJ components in other epithelial cells, E-Cad localisation was examined in the overlying epithelium. Indeed, intracellular dots of E-Cad are also observed during polarity establishment at the onset of cellularisation in Drosophila embryos, when MTs are depolymerised with colchicine (Harris and Peifer, 2005). However, during dorsal closure, injection of colcemid does not alter $\beta$-cat localisation in epithelial cells. Defects at the final step of sealing have been reported but without cellular contact defects during migration (Jankovics and Brunner, 2006). In our conditions, when Spastin was overexpressed in epidermal cells (supplementary material Fig. S3C, $\mathrm{C}^{\prime}$ ) no E-Cad dots or discontinuities in the epithelium are observed (supplementary material Fig. S3D, $\mathrm{D}^{\prime}$ ). This indicates that the MT requirement for AJ localisation during cellularisation remains essential during tracheal morphogenesis and suggests that this requirement is less important in 2D epithelia.

We quantified AJ component levels (see Materials and Methods and supplementary material Fig. S3E-J) either within whole tracheal cells or specifically at AJs of the DB (Fig. 2G,H) and of the DT (supplementary material Fig. S3K,L). MT depletion does not affect the overall levels of Par-3 and E-Cad in tracheal cells of the DB (Fig. 2G), indicating that MT function does not interfere with the balance between their synthesis and degradation. However, Par-3 and E-Cad levels are reduced apically at AJs of the DB (Fig. 2H). Similar reductions of apical Par-3 and E-Cad at AJs occur in tracheal cells of the DT (supplementary material Fig. S3K,L). These results strongly suggest that Par-3 and E-Cad reduction at AJs is independent of the intercalation state.

To conclusively demonstrate that the absence of MTs alters the dynamic equilibrium between the pool of E-Cad/Par-3 at the AJs and the cytoplasmic pool, we performed fluorescence recovery after photo-bleaching (FRAP) at the junctions of tracheal cells expressing the chimeric protein E-Cad-GFP, comprising E-Cad fused to GFP under the endogenous regulation of the E-Cad promoter (Huang et al., 2009). Fluorescence recovery in the DB of WT and Spastinoverexpressing embryos was determined. Within the time window of $739 \mathrm{~s}, 90 \%$ of the GFP signal is recovered in the WT context, whereas only $65 \%$ is recovered upon Spastin overexpression (Fig. 2I). This indicates that the MT network is required to efficiently maintain proper E-Cad dynamics at AJs.

To allow tracheal branch outgrowth, appropriate E-Cad levels at the AJs have to be tightly regulated (Shaye et al., 2008). This precise control depends on the balance between de novo synthesis and degradation of E-Cad and its internalisation and recycling from and to the apical membrane. We therefore investigated whether the intracellular E-Cad/Par-3 dots observed in the absence of MTs are intermediates in the de novo synthesis route associated with the secretory pathway or alternatively derive from endocytosis from the apical membrane. To discriminate between those two possibilities, a dominant-negative form of Rab5 (Rab5DN) that specifically blocks internalisation by preventing early endosome formation (Entchev and González-Gaitán, 2002) was overexpressed in the tracheal system. This results in a complete block in DB intercalation, visible as two parallel lines of E-Cad (Fig. 2J-J"; supplementary material Fig. S4B) (Shaye et al., 2008). We then tested whether the intracellular E-Cad dots formed in the absence of MTs are still detected when endocytosis is impaired. Rab5DN was overexpressed in tracheal cells together with Spastin and the E-Cad distribution examined at stage 14. Interestingly, intracellular E-Cad dots are still visible in tracheal cells (Fig. 2K-K"). In addition, the E-Cad-positive dots colocalise with Par-3 (Fig. 2L-L"). Taken together, these results indicate that intracellular dots of E-Cad/Par-3 derive from the de novo synthesis pathway. They also suggest that the interaction between these two proteins probably occurs during trafficking between the Golgi apparatus and their targeting to the plasma membrane.

Altogether, these results reveal an MT-dependent mechanism to apically maintain Par-3 and E-Cad levels at AJs during tubular epithelium remodelling. In addition, this mechanism appears to rely on de novo synthesis of E-Cad rather than recycling from the apical plasma membrane.

\section{Differences in E-Cad dynamics between tracheal and epidermal cells}

To further investigate the differences in E-Cad maintenance between tracheal and epidermal cells, we used the transgenic line expressing E-Cad fused to photo-convertible EosFP (Cavey et al., 2008). The distribution of the E-Cad-EosFP photo-converted pool in tracheal cells and in the epithelium (Fig. $3 \mathrm{~A}^{\prime}, \mathrm{B}^{\prime}, t=0 \mathrm{~min}$ ) is similar to that of the endogenous E-Cad-GFP (Fig. 3A,B, $t=0$ min) of stage 14 embryos. We monitored the stability of the photoconverted E-Cad-EosFP for $\sim 1 \mathrm{~h}$ and determined that reduction in the E-Cad-EosFP signal is not due to photo-bleaching (see Materials and Methods). Quantifications show two different speeds of E-Cad-EosFP signal disappearance (Fig. 3C). Furthermore, $54 \%$ of the initial signal remains in epidermal cells whereas only $23 \%$ of the initial pool is left in tracheal cells. These results emphasize that, in tracheal cells, E-Cad is highly dynamic, requiring de novo synthesis and the MT network. By contrast, in epidermal cells, E-Cad is more stable and its localisation is MT independent.

\section{Recycling endosome vesicles are apically enriched in an MT-dependent process}

We then determined how the MT network organises E-Cad intracellular trafficking so that a proper E-Cad level is maintained at AJs. The distribution of trafficking vesicles in WT and MT-depleted tracheal cells was analysed. In a WT context, Rab5-positive endocytic vesicles are distributed throughout the cytoplasm, with a weak apical bias (Fig. 4A). In the absence of MTs, this distribution is not altered (Fig. 4F) and the Rab5dependent intercalation is unaffected in the DB. Post-Golgi and late endosome vesicle localisation was also examined using antiLava lamp (Lva) and Hrs antibodies, respectively. No changes are observed in their distribution in MT-depleted tracheal cells compared with WT (Fig. 4B,C,G,H).

The localisation of recycling endosome vesicles identified with Rab11 or the Rab11-interacting protein Nuclear fallout (Nuf) was next examined. These vesicles accumulate in the apical domain of 


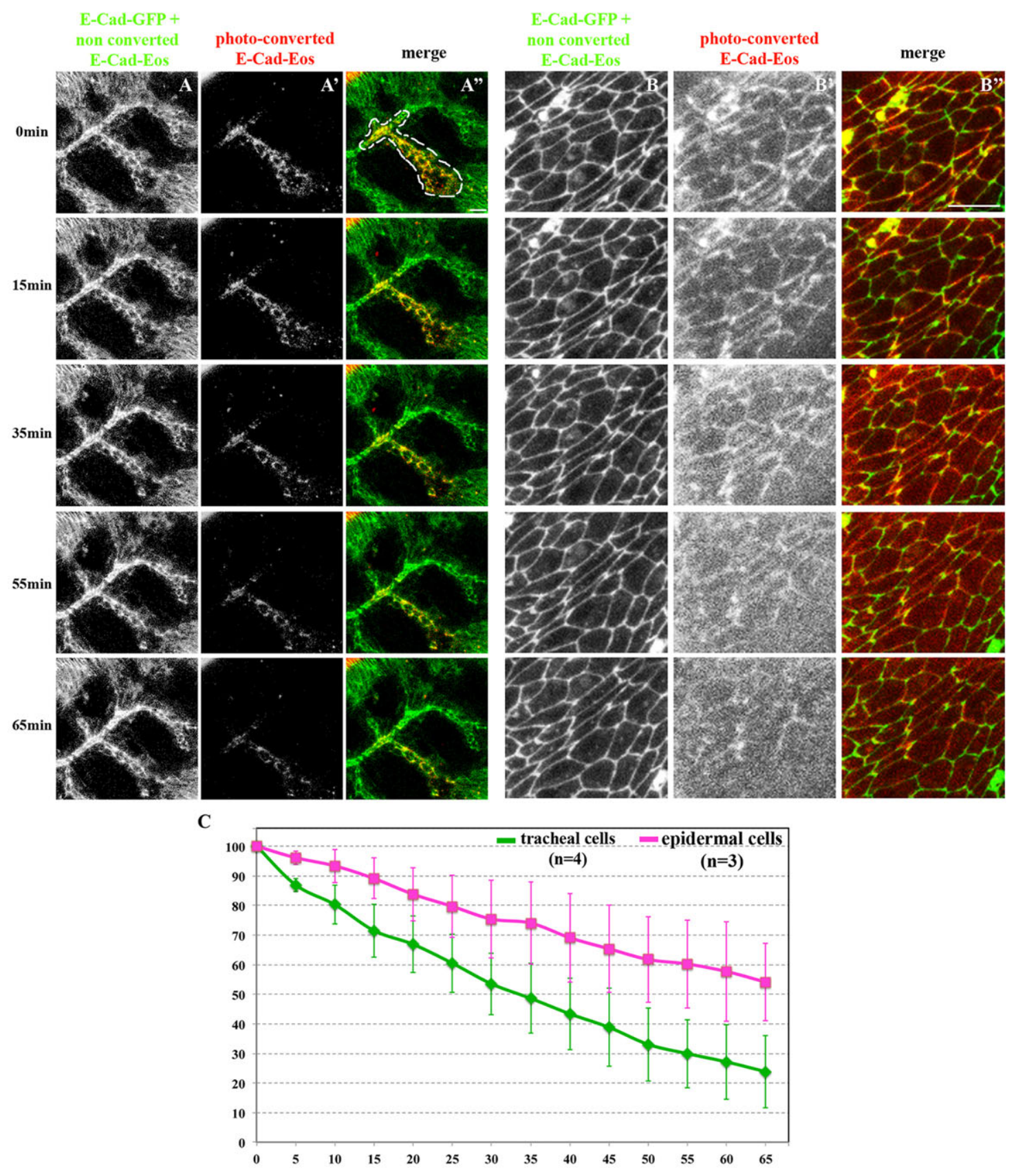

Fig. 3. E-Cad dynamics in tracheal and epidermal cells. E-Cad-GFP signal was used to define the region of interest (ROI) for the photo-conversion of E-CadEos-FP in tracheal $\left(A, A^{\prime}\right)$ and epidermal $\left(B, B^{\prime}\right)$ cells. The ROI corresponds to the outlined region in $A^{\prime \prime}$ and to the whole panel in $B^{\prime \prime}$. Note the complete absence of signal outside the ROI in $\mathrm{A}^{\prime}$. (C) Quantification of the photo-converted pool of E-Cad-EosFP over $1 \mathrm{~h}(t=0 \mathrm{~min}, 100 \%)$ in tracheal cells and epidermal cells. The two plots show significant differences $(P<0.0001$, ANOVA). Error bars indicate s.e.m. Scale bars: $10 \mu \mathrm{m}$.

WT tracheal cells (Fig. 4D,E). This asymmetric distribution is abolished in tracheal cells lacking MTs (Fig. 4I,J). The density of Nuf-positive vesicles is reduced by $60 \%$ in the absence of MTs in the apical part of the branch (Fig. 4K). We verified that the overall cellular level of recycling endosome vesicles is unaffected in the MT-depleted context (Fig. 4K). This demonstrates that the distribution of recycling endosomes is asymmetrical in tracheal cells and requires the MT network.

We checked for a potential colocalisation of E-Cad with Lva, Hrs and Nuf to test whether E-Cad accumulation is associated with particular types of trafficking vesicles, especially those involved in the de novo synthesis route. Visualising the colocalisation of E-Cad with Rab11 or Rab5 is technically impossible owing to an incompatibility in the fixation procedures. A partial colocalisation of E-Cad-positive dots with dispersed Nuf-positive vesicles is visible (Fig. 4L-M"). No robust colocalisation of E-Cad dots with Lva-positive or Hrs-positive vesicles is detected (data not shown). These results thus suggest that, in the absence of MTs, E-Cad accumulates in recycling endosome vesicles that are mispositioned within tracheal cells and cannot sustain efficient trafficking towards the apical domain. They also indicate that E-Cad intracellular vesicles, formed in the absence of MTs and emanating from the de novo synthesis pathway, pass through the Rab11-positive recycling endosome to reach the AJs. It is interesting to note that in vivo, as reported in cellular model systems, recycling endosomes can act as intermediate compartments for the post-Golgi trafficking 


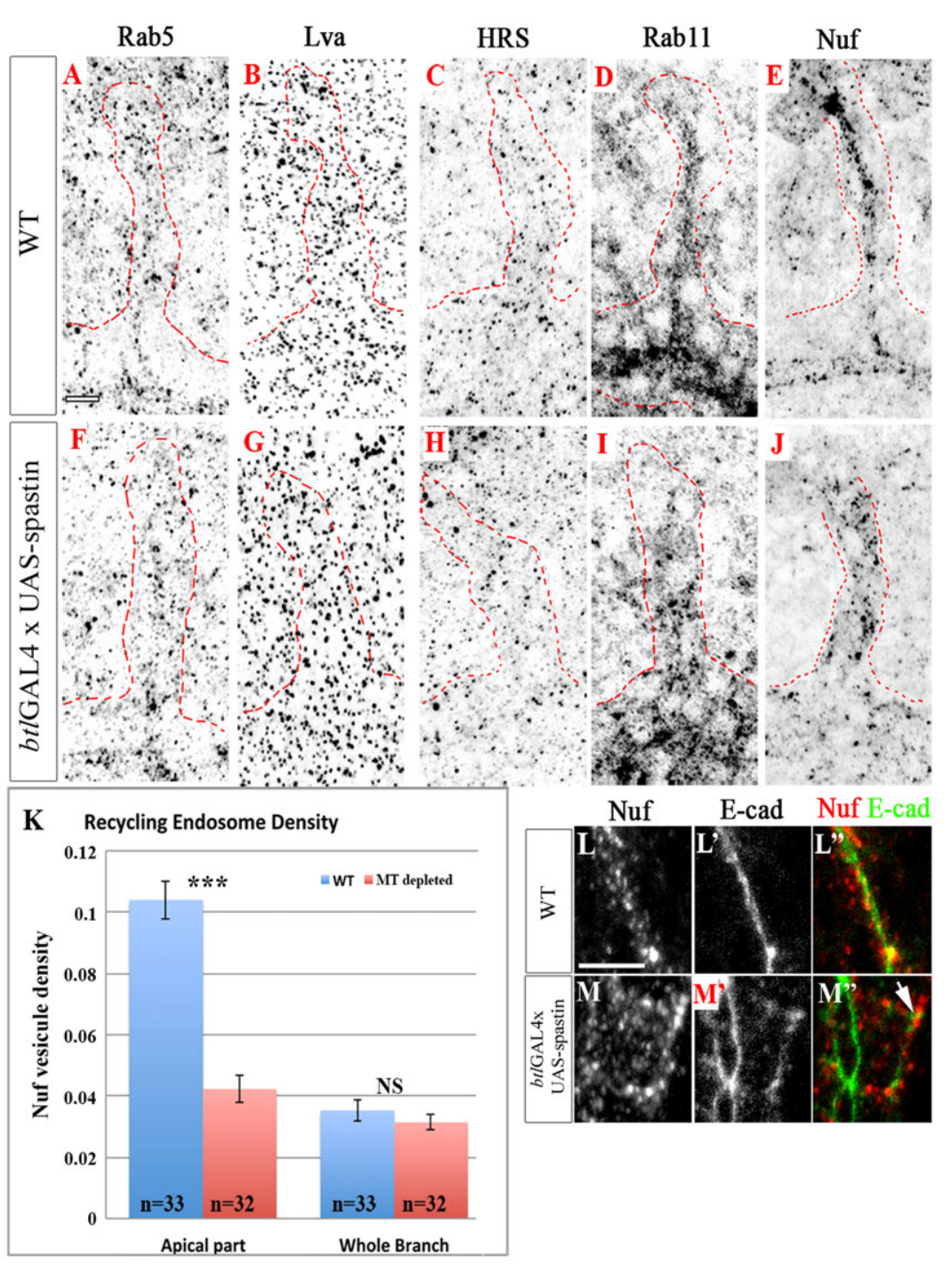

Fig. 4. Intracellular accumulation of E-Cad partially localises to the recycling endosome. (A-J) Distribution of intracellular trafficking vesicles in WT (A-E) or MT-depleted (F-J) tracheal cells of stage 14-15 embryos. Distributions of Rab5-positive endocytic vesicles (A,F), Lva-positive postGolgi vesicles $(B, G)$ and $\mathrm{Hrs}$-positive late endosome vesicles $(\mathrm{C}, \mathrm{H})$ are unchanged in MT-depleted contexts. The distribution of recycling endosome vesicles, identified by Rab11 (D,I) or Nuf $(E, J)$, is apically enriched in WT tracheal cells and appears dispersed in the absence of MTs. (K) The ratio of surface covered by apically located Nuf-positive vesicles over the DB surface apical domain or the DB total surface as measured in WT and MT-depleted contexts. Error bars indicate s.e.m.; NS, not significant; ${ }^{* \star *} P<0.001$ (Student's $t$-test). (L-M") Enlarged views of WT (L-L") or MT-depleted $\left(\mathrm{M}-\mathrm{M}^{\prime \prime}\right)$ tracheal cells of the DB. Intracellular dots of E-Cad observed in the absence of MTs partially colocalise with Nufpositive vesicles (arrow). Scale bars: $5 \mu \mathrm{m}$.

and exocytosis of E-Cad towards the plasma membrane (Rodriguez-Boulan and Macara, 2014).

\section{Apical targeting of recycling endosomes is upstream of E-Cad and Par-3 localisation}

During epithelial polarity establishment in the Drosophila embryo, Par-3 acts upstream of AJ formation as it localises properly in a $\beta$-cat mutant context (Harris and Peifer, 2004). Conversely, in the absence of Par-3, E-Cad reaches the plasma membrane but does not succeed in clustering at the future AJs (Harris and Peifer, 2004). However, during the maintenance of integrity of the dorsal thorax epithelium, Par-3 mutant cells do not exhibit mislocalised E-Cad (Georgiou et al., 2008; Leibfried et al., 2008). The relationship between Par-3 and E-Cad was thus investigated in tracheal cells. Embryos mutant for the Par-3 protein null allele $\left(b a z^{815-8}\right)$ exhibit strong tracheal defects; most DBs fail to initiate migration at stage 14 , presumably because of dorsal closure defects. We thus focused on the DT because modifications of E-Cad in the absence of MTs do not depend on the intercalation status (Fig. 2F"; supplementary material Fig. S3K,L). In MTdepleted DTs, intracellular dots of AJ components, similar to those seen in DBs, are detected (Fig. 5B,B',D,E). The loss of Par-3 does not affect E-Cad localisation in tracheal cells (Fig. 5G, $\mathrm{G}^{\prime}$ ). Similarly, in E-Cad loss-of-function mutant embryos $\left(s h g^{2}\right)$ the
Par-3 distribution profile is preserved (Fig. 5I,J). These data demonstrate that apical localisations of E-Cad and Par-3 are mutually independent in the fully polarised epithelium of the tracheal system. They also indicate that, although E-Cad and Par-3 colocalise at the level of the recycling endosome compartment (Fig. 2L), this colocalisation is not essential for their apical targeting.

Taking into account emerging evidence for reciprocal regulations between polarity proteins and membrane trafficking (Shivas et al., 2010), we tested whether Par-3 and E-Cad could also contribute to the apical accumulation of recycling endosomes in a feedback process. In the absence of MTs, Nuf apical distribution is affected in the DT, as in the DB (Fig. 5A,C). Recycling endosome distribution is unaltered in the DT of $b a z^{815-8}$ (Fig. 5F) and $s h g^{2}$ (Fig. 5H) mutant embryos, indicating that the apical accumulation of recycling endosomes is an upstream step in the pathway of E-Cad and Par-3 localisations.

\section{Recycling endosomes sustain E-Cad and Par-3 at AJs during DB extension}

Recycling endosomes are important for E-Cad targeting to AJs in cell culture and in vivo (Langevin et al., 2005; Blankenship et al., 2007; Desclozeaux et al., 2008). We tested whether recycling endosomes are necessary for proper E-Cad and Par-3 targeting at AJs in the DB. 


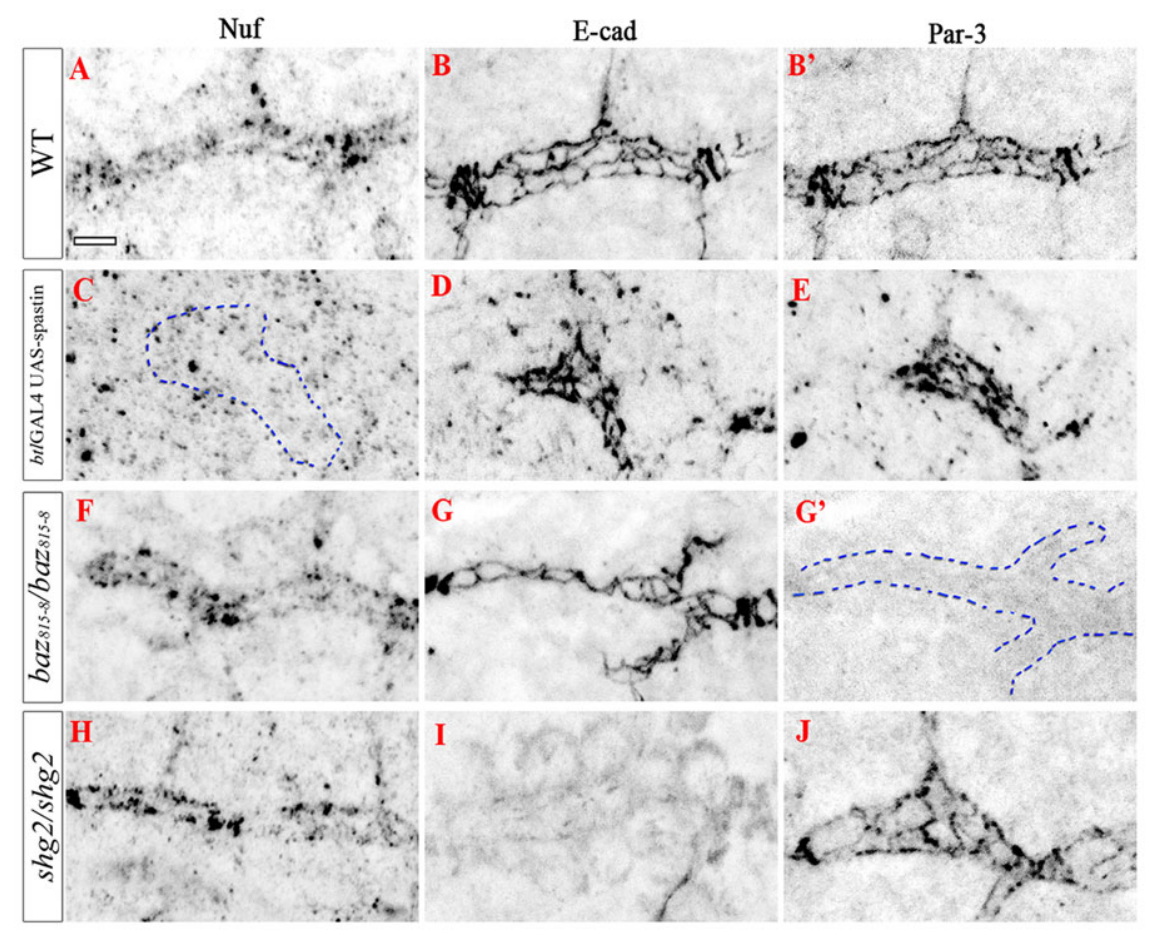

Fig. 5. Apical accumulation of recycling endosomes is upstream of E-Cad and Par-3 localisations. DT of stage 14/15 embryos showing Nuf-positive recycling vesicle distribution in WT (A), MT-depleted (C), Par-3 mutant (baz ${ }^{815-8}$ homozygote, F) or E-Cad mutant (shg ${ }^{2}$ homozygote, H). E-Cad (B,D,G,I) and Par-3 $\left(B^{\prime}, E, G^{\prime}, J\right)$ are also detected in the same genetic backgrounds. The apical enrichment of recycling endosomes is affected in MT-depleted contexts (compare $\mathrm{A}$ with $\mathrm{C}$ ). This apical enrichment is unchanged in Par-3 mutant (F) and E-Cad mutant (H) DT. Blue dashed lines highlight the DT apical part. Scale bar: $5 \mu \mathrm{m}$.

A dominant-negative form of Rab11 (Rab11DN) was overexpressed in the tracheal system (Fig. 1N). Rab11DN behaves as a dominantnegative, as the Nuf distribution is strongly reduced (supplementary material Fig. S5A,B). Rab11DN overexpression leads to phenotypes similar to those observed when MTs are depleted (Fig. 1N), inducing $37 \%$ cell overelongation and $45 \%$ branch break during DB extension (Fig. 1B). The distribution of E-Cad and Par-3 was then examined in this context (Fig. 6A-A",B-B"). Both Par-3 and E-Cad levels are significantly reduced at the AJs (Fig. 6C). Interestingly, in contrast to the effect of Spastin overexpression, E-Cad appears diffuse in the cytoplasm, where it does not localise with Par-3 (Fig. 6B"'). In epithelial cells, Rab11DN overexpression also causes a strong reduction in Nuf staining (supplementary material Fig. S6A, $\mathrm{A}^{\prime}$ ). However, it does not affect E-Cad distribution (supplementary material Fig. S6B, $\mathrm{B}^{\prime}$ ), again highlighting the differences between a $2 \mathrm{D}$ epithelium and one undergoing cell remodelling in $3 \mathrm{D}$. These results suggest that functional recycling endosomes are required for the proper Par-3 and E-Cad apical targeting that is essential for DB extension. Altogether, our data demonstrate an MT-dependent apical restriction of recycling endosomes that promotes adhesion by maintaining Par-3 and E-Cad at AJs during organogenesis.

\section{Apical distribution of AJ components and recycling endosomes requires the MT motor Dynein}

We previously showed that, after placode invagination, MTs are nucleated and anchored at the apical domain just above the AJs (Brodu et al., 2010). MT minus ends are thus located in the apical domain where Rab11-positive recycling endosomes are enriched. In addition, the Rab11 effector Nuf physically interacts with the minus-end MT motor Dynein during mitosis (Riggs et al., 2007). We thus examined whether Dynein is required for the apical enrichment of recycling endosomes and thus for the maintenance of Par-3 and E-Cad levels during branch extension.

Components of the Dynein motor have a large maternal contribution, and zygotic mutants or the expression of RNAi transgenes do not have any tracheal phenotype in embryos (data not shown). As an alternative approach, we used ciliobrevin D, a cytoplasmic Dynein inhibitor that acts in vitro as well as in live cells (Firestone et al., 2012; Łuksza et al., 2013). At stage 14, intracellular E-Cad dots are detected in treated embryos (Fig. 7B,B") in contrast to control embryos (Fig. 7A, $\mathrm{A}^{\prime \prime}$ ). The E-Cad-positive dots colocalise with Par-3 (Fig. 7B,B'). Localisation of both E-Cad and Par-3 is reduced at AJs. At stage 16, treatment with ciliobrevin D leads to phenotypes similar to those observed when MTs are depleted, with $26 \%$ branch break and $37 \%$ cell overelongation (Fig. 7C-E). In addition, the apical enrichment of Nuf-positive recycling vesicles is abolished in tracheal cells treated with ciliobrevin D (Fig. 7F, $\mathrm{F}^{\prime}, \mathrm{G}^{\prime}$ ) and vesicles are now dispersed in the cytoplasm. Similar to MT-depleted tracheal cells, a partial colocalisation of E-Cad-positive dots with dispersed Nuf-positive vesicles is observed (arrows in Fig. 7G).

Thus, depleting MTs from tracheal cells or inhibiting the MT motor protein Dynein leads to similar phenotypes during tracheal branch formation. Consequently, these results demonstrate the functional importance of an MT-Dynein-dependent process in maintaining the AJ component trafficking that is essential for tracheal morphogenesis.

\section{DISCUSSION}

We reveal the importance of the functional interplay between MTs, vesicular trafficking and the control of AJ dynamics in cells undergoing extensive remodelling through collective migration.

MT depletion in tracheal cells induces the formation of intracellular dots containing E-Cad and Par-3. Interestingly, these intracellular accumulations are only seen in remodelling tracheal cells and not in planar epithelia. These dots are still detected when endocytosis is affected, showing that they are de novo synthesis route intermediates. Altogether, this suggests that maintaining the correct level of E-Cad/Par-3 at the apical domain requires a continuous supply of newly synthesized proteins, which could be essential for the intensive AJ reorganisation that occurs during cell intercalation and elongation of the tracheal branch. 


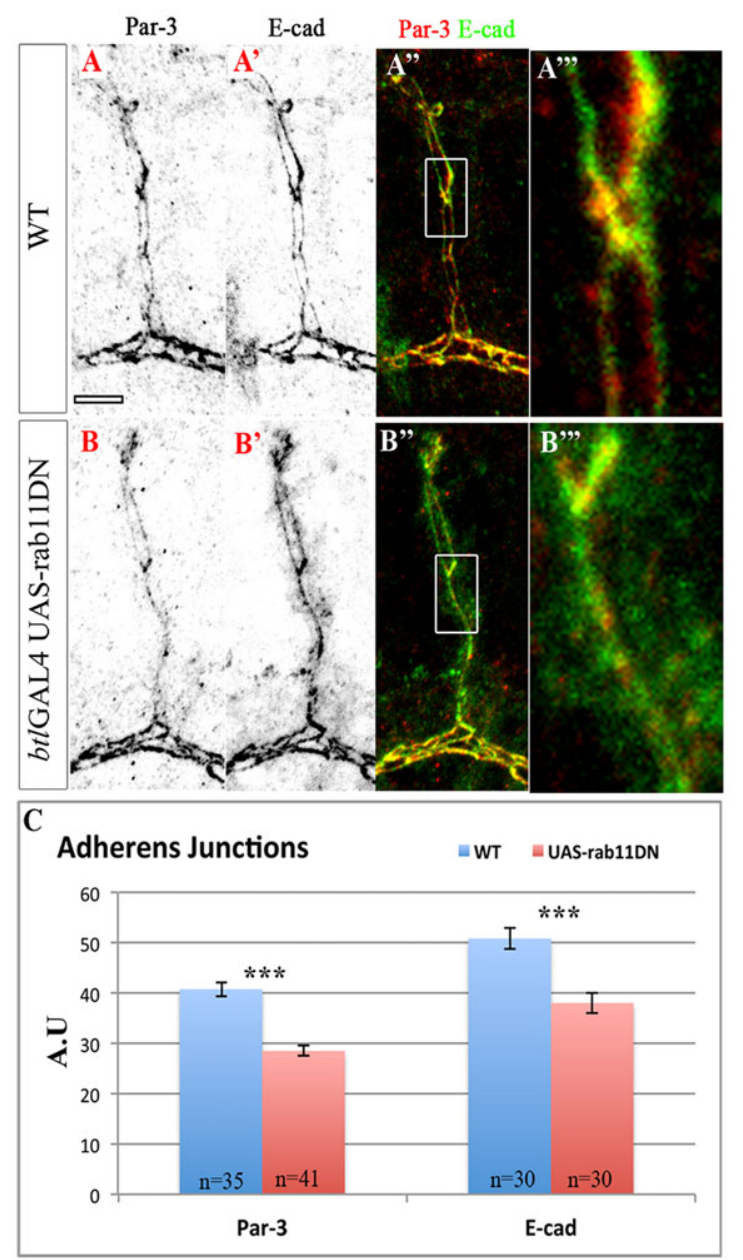

Fig. 6. Recycling endosomes sustain E-Cad and Par-3 at AJs during DB extension. (A-B"') DB of stage 14 embryos showing Par-3 $(A, B)$ and $E-C a d$ $\left(A^{\prime}, B^{\prime}\right)$ distributions in WT $\left(A-A^{\prime \prime \prime}\right)$ or when Rab11DN is overexpressed $\left(B-B^{\prime \prime \prime}\right)$. $A^{\prime \prime \prime}$ and $B^{\prime \prime \prime}$ are enlarged views of $A^{\prime \prime}$ and $B^{\prime \prime}$, respectively. Rab11DN overexpression induces a decrease in Par-3 and E-Cad apical levels. E-Cad cytoplasmic accumulation is visible, which does not colocalise with Par-3.

(C) The mean fluorescence intensity (A.U) of Par-3 and E-Cad along the AJs in WT and Rab11DN-overexpressing contexts. Error bars indicate s.e.m.; ${ }^{* * *} P<0.001$ (Student's $t$-test). Scale bar: $5 \mu \mathrm{m}$.

Using the photo-convertible E-Cad-EosFP in flat epithelium, Cavey et al. showed that E-Cad that is engaged in homophilic interactions at the AJs forms very stable domains (Cavey et al., 2008). We demonstrate that MT depletion does not affect the integrity of this 2D epithelium. In addition, we show that the pool of photo-converted E-Cad-EosFP is less stable in tracheal cells than in epidermal cells. Together with our FRAP assay suggesting that AJs are more dynamic in tracheal cells than in epithelial cells (see below), our results highlight a specific fine-tuning of AJ components in tracheal cells undergoing cell movement and cell shape changes in 3D through cell intercalation, cell elongation and thereby organ formation. This fine-tuning is likely to cycle between internalisation, recycling, degradation and de novo synthesis, the latter being MT dependent. When the balance is altered in the absence of MTs, and thus when E-Cad or Par-3 are reduced at AJs, tracheal cells overelongate after completing intercalation. During branch elongation without MTs, the two migrating leading cells generate a pulling force on the following stalk cells, which display a critical reduction in AJ components.

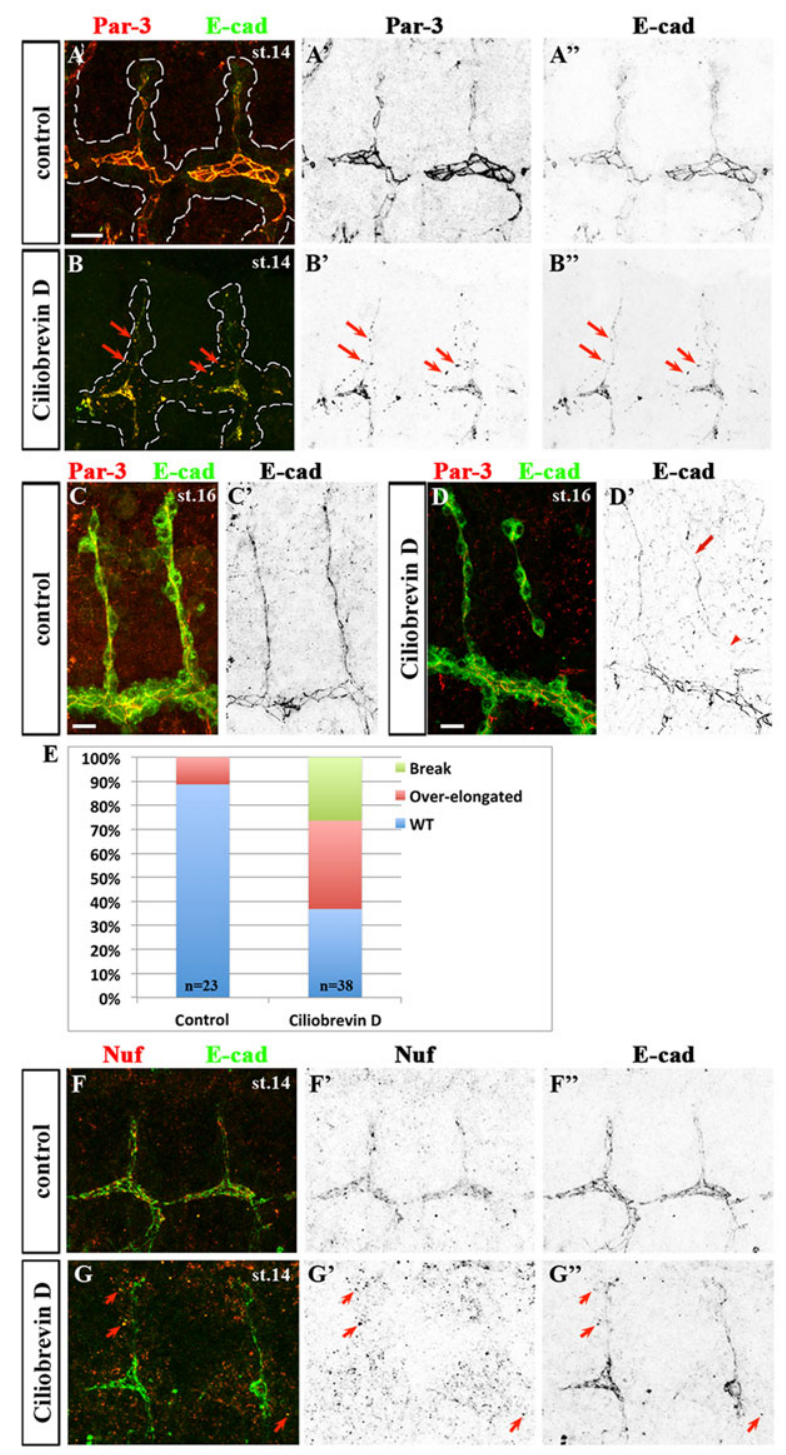

Fig. 7. Apical enrichment of recycling endosomes and distribution of E-Cad and Par-3 at AJs depend on Dynein. CD8::GFP expression in tracheal cells of untreated $(A, C, F)$ or ciliobrevin $D$-treated $(B, D, G)$ embryos at stage $14(A, B, F, G)$ or stage $15(C, D)$. Apical localisation of Par-3 $\left(B^{\prime}\right)$ and $\mathrm{E}-\mathrm{Cad}\left(\mathrm{B}^{\prime \prime}\right)$ is reduced in ciliobrevin D-treated embryos as compared with untreated embryos $\left(A^{\prime}, A^{\prime \prime}\right)$. In addition, Par-3 and E-Cad form large cytoplasmic accumulations in treated embryos $\left(\mathrm{B}^{\prime}, \mathrm{B}^{\prime \prime}\right.$, arrows). Cell overelongation (arrow) and branch break (arrowhead) are visible as of stage 16 in treated embryos $\left(D, D^{\prime}\right)$ as compared with controls $\left(C, C^{\prime}\right)$. (E) Percentage of WT, overelongated or broken DBs in untreated or ciliobrevin D-treated embryos at stage 16. (F-G") Nuf-positive recycling endosome vesicles are apically enriched in controls $\left(F^{\prime}\right)$ and are dispersed in treated embryos $\left(G^{\prime}\right)$. They now partially colocalise with intracellular dots of E-Cad (G,G", arrows). Scale bars: $10 \mu \mathrm{m}$.

Either the tip cell or the base cell of the stalk becomes unable to maintain its integrity in response to this force. Consequently, this tracheal cell overelongates by a factor 1.8 , preventing the remaining stalk cells from reaching their average size. As a result, DBs present a single overelongated cell and several underelongated cells.

We detected an overlap between the E-Cad intracellular dots generated in the absence of MTs and recycling endosome vesicles. We also showed that interfering with Rab11 function in tracheal cells induces cell overelongation and affects E-Cad and Par-3 
distribution at the AJs, as does MT depletion. These results illustrate that the E-Cad de novo synthesis pathway passes through the Rab11positive recycling endosome compartment. The overlap of E-Cad and recycling endosome markers represents only a small proportion of the total intracellular E-Cad, suggesting a transient residence in this vesicular compartment for this newly synthesized protein (Langevin et al., 2005; Desclozeaux et al., 2008). Recent studies conducted in different model systems have revealed that some newly synthesized apical plasma membrane proteins, such as E-Cad and Rhodopsin, leave the trans-Golgi network to cross Rab11-positive recycling endosome compartments before reaching the apical surface (Brand and Perrimon, 1993; Satoh et al., 2005; Desclozeaux et al., 2008; Rodriguez-Boulan and Macara, 2014). We unveil that this apical trafficking route is used specifically in tracheal cells and requires the MT network. Quantification of Rab11DN-associated defects upon tracheal branch formation reveals that impairing recycling endosome function has a similar effect to altering the distribution of E-Cad at AJs by depleting the MT network. Moreover, interfering with Rab11 function reduces Par-3 levels at the AJs of tracheal cells. Interestingly, Par-3 does not colocalise with the E-Cad cytoplasmic pool, indicating that functional recycling endosomes are required by Par-3 and E-Cad to assemble as a complex and to be targeted to the apical domain of tracheal cells. However, as E-Cad and Par-3 can be apically targeted in the absence of the other, this suggests that apical targeting of E-Cad and Par-3 can be independent in tracheal cells or that redundant pathways could sustain the localisation of each protein. For example, the Nectin protein Echinoid is required for Par-3 localisation at AJs in shg mutant cells (Wei et al., 2005). Moreover, the apical distribution of $\mathrm{PI}(4,5) \mathrm{P} 2$ in the Drosophila follicular epithelium sustains Par-3 apical anchorage at the plasma membrane (Claret et al., 2014). Furthermore, Par-3-independent localisation of E-Cad has been observed (Georgiou et al., 2008; Leibfried et al., 2008).

Dynamic MTs in the ectoderm locally upregulate AJ turnover through RhoA activity (Bulgakova et al., 2013). RhoA stabilises cellular contacts through acto-myosin regulation. In tracheal cells, MT depletion does not alter actin distribution. Moreover, tracheal cells mutant for the Myosin light chain zipper (zip) and also expressing a dominant-negative form of Zip do not present obvious defects in E-Cad distribution at stage 14 (data not shown). By contrast, MT depletion induces the cytoplasmic accumulation of E-Cad and Par-3 in tracheal cells only and not in ectodermal cells at the same developmental stage. Thus far, cytoplasmic accumulation of E-Cad and Par-3 has only been observed after colchicine-induced MT depolymerisation during polarity establishment at embryo cellularisation (Harris and Peifer, 2005), when AJs are extremely dynamic (Huang et al., 2011) and vesicular trafficking is strongly active (Lecuit, 2004). We demonstrate that the E-Cad distribution in tracheal cells is more sensitive to MT depolymerisation and to Rab11DN overexpression than that in the overlying ectodermal cells. The comparison of the maximum recovery of the E-Cad signal in a FRAP assay in tracheal cells (our data) and in ectodermal cells (Bulgakova et al., 2013), together with the differences in stability of the photo-converted E-Cad-EosFP between these two tissues, suggest that AJs are more dynamic in tracheae. It is thus conceivable that tracheal cells, which undergo extensive cell shape changes through collective cell migration, require more dynamic AJs as sustained by an efficient targeting of E-Cad. These discrepancies between ectodermal and tracheal epithelia underline the importance of investigating MT function in different morphogenetic contexts under different constraints.
We demonstrate that the MT minus-end motor Dynein is essential for the restricted localisation of recycling endosomes in a developing organism. The Dynein requirement for the apical enrichment of recycling endosomes is in agreement with the MT minus ends being anchored at the apical plasma membrane (Brodu et al., 2010). The asymmetric distribution of recycling endosome vesicles has been observed in various differentiated cell types, especially during cell division (Goldenring et al., 1996; Emery et al., 2005; Riggs et al., 2007). Vesicles are found enriched either in the apical domain or at the microtubule-organising centre (MTOC; i.e. the centrosome) during mitosis. Indeed, in vivo, Dynein physically interacts with Nuf. During metaphase, Dynein is required for the maintenance of Nuf at the centrosome (Riggs et al., 2007). Here we demonstrate that Dynein is also required for the apical distribution of recycling endosomes in non-dividing tracheal cells (Fig. 7). In a context in which Dynein function is altered, Nuf-positive recycling endosome vesicles are dispersed but colocalise with E-Cad and Par-3 intracellular dots, indicating that the recycling endosome compartment remains functional for the assembly of such a complex.

We have previously characterised the relocalisation of the MTOC in tracheal cells and have shown that MTs are nucleated and anchored at the apical domain just above the AJs. We have also demonstrated that such MT organisation is crucial for tracheal morphogenesis (Brodu et al., 2010). Non-centrosomal MT organisation occurs in many differentiated cell types but the functional relevance of such an organisation is still poorly understood. It will be informative to investigate whether such non-centrosomal MT organisation provides a means to regulate epithelial remodelling by controlling the apical enrichment of recycling endosomes and thus AJ dynamics.

\section{MATERIALS AND METHODS}

\section{Drosophila strains}

Fly lines are described in the supplementary Materials and Methods.

\section{Immunohistochemistry and data acquisition}

Embryos were processed as described (Brodu et al., 2010; Brodu, 2004). For further details of fixation and immunostaining, see supplementary Materials and Methods.

\section{Live imaging}

Imaging of EB1-GFP comets and of tracheal development in the absence of MTs is described in the supplementary Materials and Methods.

\section{Fluorescence recovery after photo-bleaching}

WT and $b t l$-GAL4 $\times$ UAS-Spastin embryos, in which the only source of E-Cad is an E-Cad-GFP knock-in construct, were analysed. Embryos were imaged at $25^{\circ} \mathrm{C}$ using an argon laser $(488 \mathrm{~nm})$ and $\times 63 / 1.4$ NA oilimmersion lens on a Zeiss LSM 700 confocal microscope. Pre- and postbleach images were $512 \times 512$ pixels $(1$ pixel $=132 \mathrm{~nm}$ ), with a $5.2 \mathrm{~s}$ frame rate and no delay between consecutive images. The bleached areas correspond to two parallel lines of intercellular junctions in the DB. This area covers half of the junction length. After two pre-bleach scan acquisition, fluorescence recovery was monitored over at least $770 \mathrm{~s}$ on projections of two consecutive stacks with a step of $1.8 \mu \mathrm{m}$. The StackReg plugin in Image J (NIH) was used to correct for movements. As a control region of interest (ROI), intercellular junctions formed by adjacent tracheal cells were used to normalise fluorescence recovery.

\section{Photo-conversion of E-Cad-EosFP}

Photo-conversion of E-Cad-EosFP was performed on a Zeiss LSM 780 confocal microscope, iterating 70 times a pulse of a $405 \mathrm{~nm}$ laser at $100 \%$ power over ROIs of variable size. Non-converted E-Cad-EosFP and E-CadGFP were excited at $488 \mathrm{~nm}$ and photo-converted E-Cad-EosFP was excited 
at $543 \mathrm{~nm}$. The objective used was a $\times 63$ oil immersion, 1.4 NA. Turnover of E-Cad in tracheal and epidermal cells of stage 14 embryos was inferred by measuring variations of photo-converted signal intensities within the ROIs over a $1 \mathrm{~h}$ period with acquisition every $5 \mathrm{~min}$. Values were normalised with $t=0$ (the time of photo-conversion) set to $100 \%$. To exclude that photobleaching contributes to the decrease in signal intensity, another ROI within the same embryo was photo-converted at $t=0$, and signal intensity was measured after $1 \mathrm{~h}$.

\section{Image processing, quantification and statistics}

Images of fixed embryos were acquired with a Zeiss LSM 700 confocal microscope. The luminal marker Uif defines an apical mask corresponding to the tracheal cell apical side. UAS-CD8::GFP expression under the $b t l$-GAL4 driver defines a mask corresponding to the cell volume of the DB. Apical and whole branch masks were used to create ROIs for each $z$-step. Original $z$-stack acquisitions were normalised and transformed into binary images. Signals were processed with the Fill holes and Dilate options (count: 3; iteration: 6) in order to include AJs for E-Cad and Par-3 stainings and subapical recycling endosomes for Nuf staining.

The mean of intensity signals at AJs or within Nuf-positive vesicles was calculated by drawing lines in normalised WT $z$-stacks. This mean was used as a cut-off threshold for detected signals. Arbitrary unit (A.U.) corresponds to immunofluorescence levels within $z$-stacks of the ROI according to our acquisition set up on Zeiss LSM 700 confocal. Nuf vesicles correspond to the threshold signal and at least three pixels in contact correspond to $0.072 \mu \mathrm{m}^{2}$. For Rab11DN overexpression, three lines were drawn within the AJs and A.U. corresponds to the mean of this signal intensity.

\section{Drug treatment}

Embryos expressing the membrane-bound form CD8::GFP in tracheal cells were collected, dechorionated with bleach and transferred to tubes containing a 1:1 mixture of n-heptane and Drosophila Schneider's Medium (Gibco) and either $50 \mu \mathrm{M}$ ciliobrevin D (Calbiochem) dissolved in DMSO or just DMSO as control. After $1 \mathrm{~h}$ incubation with gentle shaking at room temperature, $n$-heptane was removed and embryos were incubated in Schneider's Medium containing $50 \mu \mathrm{M}$ ciliobrevin D or just DMSO for an additional 2 hours. Embryos were then fixed and stained following standard protocols.

\section{Acknowledgements}

We thank the Institut Jacques Monod imaging facilities, J. P. Grossier and F. Perez (Institut Curie) for assistance with Image J macros and advice; H. Bellen, K. Broadie, D. Brunner, J. Casanova, M. Gonzalez-Gaitan, W. Sullivan, U. Tepass, R. Ward and A. Wodarz for reagents and Drosophila strains; the Bloomington Stock Center for fly stocks; the Developmental Studies Hybridoma Bank for monoclonal antibodies; and C. Caine, J. Casanova, A.-L. Haenni, K. Legent and J.-A. Lepesant for discussion and critical comments on the manuscript. V.B. is indebted to J. Casanova and Z. Chamoun for constant support and interest in the course of this study.

\section{Competing interests}

The authors declare no competing financial interests.

\section{Author contributions}

P.-M.L.D. and V.B. conducted the experiments and together with A.G. interpreted the data. S.C. contributed to the quantitative imaging analysis. V.B. designed the study and wrote the manuscript. All authors were involved in the final stages of writing the article.

\section{Funding}

P.-M.L.D. was supported by a fellowship from the Ministry of Research and Technology (MRT) and by a fellowship from Fondation ARC pour la Recherche sur le Cancer. This work was supported by ARC [SL220100601358 and SLR20130607102].

\section{Supplementary material}

Supplementary material available online at

http://dev.biologists.org/lookup/suppl/doi:10.1242/dev.113472/-/DC1

\section{References}

Akhmanova, A. and Steinmetz, M. O. (2008). Tracking the ends: a dynamic protein network controls the fate of microtubule tips. Nat. Rev. Mol. Cell Biol. $\mathbf{9}$, 309-322.
Blankenship, J. T., Fuller, M. T. and Zallen, J. A. (2007). The Drosophila homolog of the Exo84 exocyst subunit promotes apical epithelial identity. J. Cell Sci. 120, 3099-3110.

Brand, A. H. and Perrimon, N. (1993). Targeted gene expression as a means of altering cell fates and generating dominant phenotypes. Development 118, 401-415

Brieher, W. M. and Yap, A. S. (2013). Cadherin junctions and their cytoskeleton(s). Curr. Opin. Cell Biol. 25, 39-46.

Brodu, V., Elstob, P. R. and Gould, A. P. (2004). EGF receptor signaling regulates pulses of cell delamination from the Drosophila ectoderm. Dev. Cell 7, 885-895.

Brodu, V., Baffet, A. D., Le Droguen, P.-M., Casanova, J. and Guichet, A. (2010). A developmentally regulated two-step process generates a noncentrosomal microtubule network in Drosophila tracheal cells. Dev. Cell 18, 790-801.

Bryant, D. M. and Mostov, K. E. (2008). From cells to organs: building polarized tissue. Nat. Rev. Mol. Cell Biol. 9, 887-901.

Bulgakova, N. A., Grigoriev, I., Yap, A. S., Akhmanova, A. and Brown, N. H. (2013). Dynamic microtubules produce an asymmetric E-cadherin-Bazooka complex to maintain segment boundaries. J. Cell Biol. 201, 887-901.

Caussinus, E., Colombelli, J. and Affolter, M. (2008). Tip-cell migration controls stalk-cell intercalation during Drosophila tracheal tube elongation. Curr. Biol. 18, 1727-1734.

Cavey, M. and Lecuit, T. (2009). Molecular bases of cell-cell junctions stability and dynamics. Cold Spring Harb. Perspect. Biol. 1, a002998.

Cavey, M., Rauzi, M., Lenne, P.-F. and Lecuit, T. (2008). A two-tiered mechanism for stabilization and immobilization of E-cadherin. Nature 453, 751-756.

Chausovsky, A., Bershadsky, A. D. and Borisy, G. G. (2000). Cadherin-mediated regulation of microtubule dynamics. Nat. Cell Biol. 2, 797-804.

Chen, X., Kojima, S.-i., Borisy, G. G. and Green, K. J. (2003). p120 catenin associates with kinesin and facilitates the transport of cadherin-catenin complexes to intercellular junctions. J. Cell Biol. 163, 547-557.

Claret, S., Jouette, J., Benoit, B., Legent, K. and Guichet, A. (2014). PI(4,5)P2 produced by the PI4P5K SKTL controls apical size by tethering PAR-3 in Drosophila epithelial cells. Curr. Biol. 24, 1071-1079.

Desclozeaux, M., Venturato, J., Wylie, F. G., Kay, J. G., Joseph, S. R., Le, H. T. and Stow, J. L. (2008). Active Rab11 and functional recycling endosome are required for E-cadherin trafficking and lumen formation during epithelial morphogenesis. Am. J. Physiol. Cell Physiol. 295, C545-C556.

Emery, G., Hutterer, A., Berdnik, D., Mayer, B., Wirtz-Peitz, F., Gaitan, M. G. and Knoblich, J. A. (2005). Asymmetric Rab11 endosomes regulate delta recycling and specify cell fate in the Drosophila nervous system. Cell 122, 763-773.

Entchev, E. V. and González-Gaitán, M. A. (2002). Morphogen gradient formation and vesicular trafficking. Traffic 3, 98-109.

Firestone, A. J., Weinger, J. S., Maldonado, M., Barlan, K., Langston, L. D., O'Donnell, M., Gelfand, V. I., Kapoor, T. M. and Chen, J. K. (2012). Smallmolecule inhibitors of the AAA+ ATPase motor cytoplasmic dynein. Nature $\mathbf{4 8 4}$ $125-129$

Georgiou, M., Marinari, E., Burden, J. and Baum, B. (2008). Cdc42, Par6, and aPKC regulate Arp2/3-mediated endocytosis to control local adherens junction stability. Curr. Biol. 18, 1631-1638.

Gervais, L., Lebreton, G. and Casanova, J. (2012). The making of a fusion branch in the Drosophila trachea. Dev. Biol. 362, 187-193.

Goldenring, J. R., Smith, J., Vaughan, H. D., Cameron, P., Hawkins, W. and Navarre, J. (1996). Rab11 is an apically located small GTP-binding protein in epithelial tissues. Am. J. Physiol. 270, G515-G525.

Harris, T. J. C. and Peifer, M. (2004). Adherens junction-dependent and -independent steps in the establishment of epithelial cell polarity in Drosophila. J. Cell Biol. 167, 135-147.

Harris, T. J. C. and Peifer, M. (2005). The positioning and segregation of apical cues during epithelial polarity establishment in Drosophila. J. Cell Biol. 170, 813-823.

Huang, J., Zhou, W., Dong, W., Watson, A. M. and Hong, Y. (2009). Directed, efficient, and versatile modifications of the Drosophila genome by genomic engineering. Proc. Natl. Acad. Sci. USA 106, 8284-8289.

Huang, J., Huang, L., Chen, Y.-J., Austin, E., Devor, C. E., Roegiers, F. and Hong, Y. (2011). Differential regulation of adherens junction dynamics during apical-basal polarization. J. Cell Sci. 124, 4001-4013.

Janke, C. and Bulinski, J. C. (2011). Post-translational regulation of the microtubule cytoskeleton: mechanisms and functions. Nat. Rev. Mol. Cell Biol. 12, 773-786

Jankovics, F. and Brunner, D. (2006). Transiently reorganized microtubules are essential for zippering during dorsal closure in Drosophila melanogaster. Dev. Cell 11, 375-385.

Langevin, J., Morgan, M. J., Rossè, C., Racine, V., Sibarita, J.-B., Aresta, S. Murthy, M., Schwarz, T., Camonis, J. and Bellaïche, Y. (2005). Drosophila exocyst components Sec5, Sec6, and Sec15 regulate DE-Cadherin trafficking from recycling endosomes to the plasma membrane. Dev. Cell 9, 365-376.

Lecuit, T. (2004). Junctions and vesicular trafficking during Drosophila cellularization. J. Cell Sci. 117, 3427-3433. 
Leibfried, A., Fricke, R., Morgan, M. J., Bogdan, S. and Bellaiche, Y. (2008). Drosophila Cip4 and WASp define a branch of the Cdc42-Par6-aPKC pathway regulating E-cadherin endocytosis. Curr. Biol. 18, 1639-1648.

Łuksza, M., Queguigner, I., Verlhac, M.-H. and Brunet, S. (2013). Rebuilding MTOCs upon centriole loss during mouse oogenesis. Dev. Biol. 382, 48-56.

Meng, W., Mushika, Y., Ichii, T. and Takeichi, M. (2008). Anchorage of microtubule minus ends to adherens junctions regulates epithelial cell-cell contacts. Cell 135, 948-959.

Nejsum, L. N. and Nelson, W. J. (2007). A molecular mechanism directly linking E-cadherin adhesion to initiation of epithelial cell surface polarity. J. Cell Biol. 178, 323-335.

Ratheesh, A., Gomez, G. A., Priya, R., Verma, S., Kovacs, E. M., Jiang, K., Brown, N. H., Akhmanova, A., Stehbens, S. J. and Yap, A. S. (2012). Centralspindlin and $\alpha$-catenin regulate Rho signalling at the epithelial zonula adherens. Nat. Cell Biol. 14, 818-828.

Ribeiro, C., Neumann, M. and Affolter, M. (2004). Genetic control of cell intercalation during tracheal morphogenesis in Drosophila. Curr. Biol. 14, 2197-2207.

Riggs, B., Fasulo, B., Royou, A., Mische, S., Cao, J., Hays, T. S. and Sullivan, W. (2007). The concentration of Nuf, a Rab11 effector, at the microtubule-organizing center is cell cycle regulated, dynein-dependent, and coincides with furrow formation. Mol. Biol. Cell 18, 3313-3322.

Rodriguez-Boulan, E. and Macara, I. G. (2014). Organization and execution of the epithelial polarity programme. Nat. Rev. Mol. Cell Biol. 15, 225-242.

Satoh, A. K., O'Tousa, J. E., Ozaki, K. and Ready, D. F. (2005). Rab11 mediates post-Golgi trafficking of rhodopsin to the photosensitive apical membrane of Drosophila photoreceptors. Development 132, 1487-1497.
Shaye, D. D., Casanova, J. and Llimargas, M. (2008). Modulation of intracellular trafficking regulates cell intercalation in the Drosophila trachea. Nat. Cell Biol. 10, 964-970.

Shivas, J. M., Morrison, H. A., Bilder, D. and Skop, A. R. (2010). Polarity and endocytosis: reciprocal regulation. Trends Cell Biol. 20, 445-452.

Stehbens, S. J., Paterson, A. D., Crampton, M. S., Shewan, A. M., Ferguson, C., Akhmanova, A., Parton, R. G. and Yap, A. S. (2006). Dynamic microtubules regulate the local concentration of E-cadherin at cell-cell contacts. J. Cell Sci. 119, 1801-1811.

Teng, J., Rai, T., Tanaka, Y., Takei, Y., Nakata, T., Hirasawa, M., Kulkarni, A. B. and Hirokawa, N. (2005). The KIF3 motor transports N-cadherin and organizes the developing neuroepithelium. Nat. Cell Biol. 7, 474-482.

Uv, A., Cantera, R. and Samakovlis, C. (2003). Drosophila tracheal morphogenesis: intricate cellular solutions to basic plumbing problems. Trends Cell Biol. 13, 301-309.

Waterman-Storer, C. M., Salmon, W. C. and Salmon, E. D. (2000). Feedback interactions between cell-cell adherens junctions and cytoskeletal dynamics in newt lung epithelial cells. Mol. Biol. Cell 11, 2471-2483.

Wei, S.-Y., Escudero, L. M., Yu, F., Chang, L.-H., Chen, L.-Y., Ho, Y.-H., Lin, C.-M., Chou, C.-S., Chia, W., Modolell, J. et al. (2005). Echinoid is a component of adherens junctions that cooperates with DE-Cadherin to mediate cell adhesion. Dev. Cell 8, 493-504.

Wirtz-Peitz, F. and Zallen, J. A. (2009). Junctional trafficking and epithelial morphogenesis. Curr. Opin. Genet. Dev. 19, 350-356.

Yap, A. S., Stevenson, B. R., Abel, K. C., Cragoe, E. J., Jr and Manley, S. W. (1995). Microtubule integrity is necessary for the epithelial barrier function of cultured thyroid cell monolayers. Exp. Cell Res. 218, 540-550. 


\section{Supplementary Materials and Methods}

\section{Drosophila strains}

A description of most of the genetic elements can be found at http://flybase.net (FlyBase Consortium, 1999). Drosophila stocks and crosses were kept under standard conditions. The

following mutations were used: $s h g^{2}, s h g^{119}, b a z^{815.8}$. FM7-ftz-lacZ, CyO-wg-lacZ, TM3-ftzlacZ, or TM6-Ubx-lacZ blue balancers were used to identify homozygous embryos. The GAL4 system (Brand and Perrimon, 1993) was used for misexpression experiments. The epidermal en-GAL4 driver (A. Brand, Wellcome/CRC Institute, Cambridge, UK) and the tracheal btl-GAL4 driver (Shiga et al., 1996) were used at $29^{\circ} \mathrm{C}$ in combination with UASSpastin-GFP (Trotta et al., 2004), UAS-Spastin (our stock), UAS-EB1-GFP (Jankovics and Brunner, 2006), UAS-CD8-GFP, btl-RFP1-Moesin (Ribeiro et al., 2004) and at $18^{\circ} \mathrm{C}$ in combination with UAS-Rab11 $1^{\mathrm{S} 25 \mathrm{~N}}$ as Rab11 dominant negative and at $29^{\circ} \mathrm{C}$ in combination with UAS-Rab5 ${ }^{\mathrm{S} 43 \mathrm{~N}}$ as Rab5 dominant negative (both provided by Marcos González-Gaitán). The E-Cad-GFP has been described in (Huang et al., 2009) and the E-Cad-EosFP has been described in(Cavey et al., 2008).

\section{Immunohistochemistry}

Embryos were staged as described in (Campos-Ortega and Hartenstein, 1985) fixed and stained following standard protocols. To visualize MTs, embryos were fixed as previously described (Brodu et al., 2010). Embryos were heat-fixed and post-fixed in methanol for immunostaining using anti-Rab5 and anti-Rab11 antibodies (Miller et al., 1989). Primary antibodies were as described in (Brodu et al., 2010) with the following additions: antiarmadillo (1/500, DSHB), anti E-Cad (1/50, DSHB), anti-Crumbs (1/1000, U. Tepass), antiBazooka (1/2000, A. Wodarz), anti-Rab5 (1/50, M. Gonzalez-Gaitan); anti-Rab11 (1/100, BD Biosciences, \#610656), anti-Nuf (1/1000, W. Sullivan;), anti-Uninflatable (Uif) (1/300, R. Ward;), anti-HRS (1/500, H. Bellen;). Secondary antibodies conjugated with Cy3, Cy5 (Jackson Laboratories) or Alexa Fluor 488, 546 and 647 (Life technologies) were used.

\section{Live imaging}

Embryos were processed as described in (Brodu et al., 2004). Imaging of EB1-GFP comets was carried out using a spinning disk $491 \mathrm{~nm}$ laser $\times 100 / 1.4$ NA objective. Acquisition was every $1500 \mathrm{msec}$. Max intensity projection of $\mathrm{Z}$ stacks over $60 \mathrm{sec}$ intervals are shown. EB1GFP comets were observed in WT and Spastin overexpression contexts. For comparison, both 
genetic contexts carried the same number of UAS transgenes so that the amount of GAL4 available to drive the UAS-EB1-GFP construct was identical.

Imaging of the tracheal development in absence of MTs was performed in embryos overexpressing Spastin-GFP in the tracheal system together with the expression of btl-mRFPMoesin to highlight the tracheal apical domain (Ribeiro et al., 2004). Images were collected from stage 13-14 embryos on a Leica TCS SP5 confocal microscope with a $\times 63$ oil immersion objective, 1.4 NA using the Leica TCS NT software. 30 sections spaced by $1 \mu \mathrm{m}$ were recorded every 5min. TIFF images were processed, with ImageJ. 


\section{Supplementary Movies}

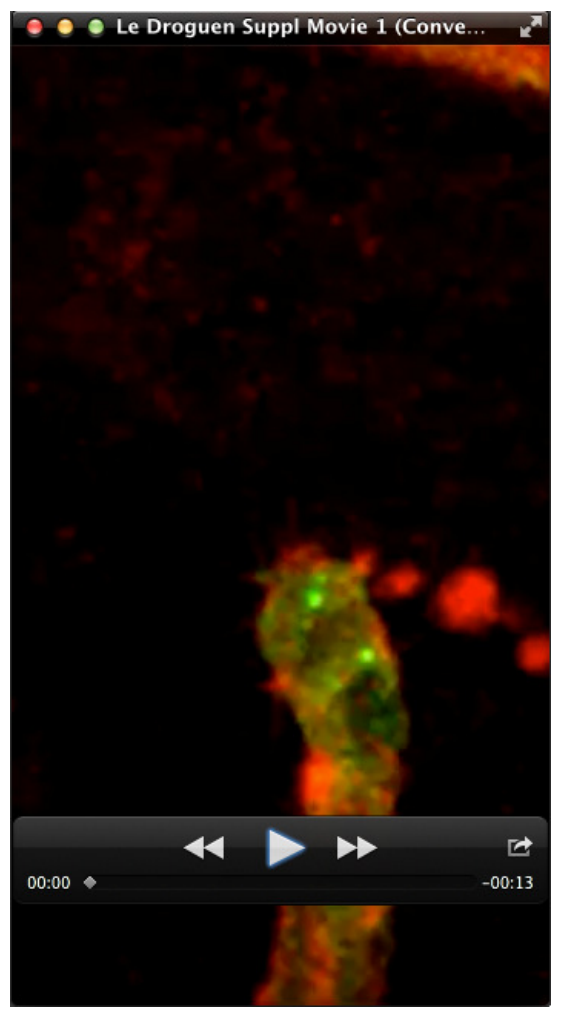

\section{Supplementary Movie 1}

Confocal life imaging of one DB expressing btl-RFP1-Moesin and UAS-spastin-GFP driven by btl-GAL4 during stages 14 to 15 . Two channels, corresponding to spastin-GFP (green) and RFP-Moesin (red), were acquired simultaneously for better time resolution. Lateral views (zstack maximum projections) were taken at $5 \mathrm{~min}$ intervals from stage 13 to 15 . Anterior is to the left. Tracheal cell at the tip of the stalk gradually over-elongates, forming a long cytoplasmic thread. The following tracheal cells are under-elongated (white arrows). 


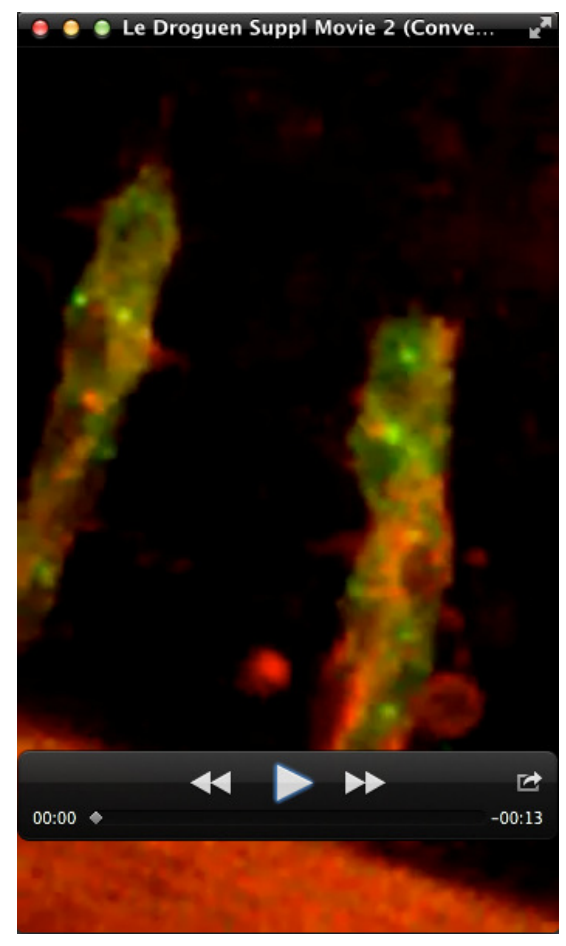

\section{Supplementary Movie 2}

Confocal life imaging of DB expressing btl-RFP1-Moesin and UAS-spastin-GFP driven by btl-GAL4 during stages 14 to 15 . Two channels, corresponding to spastin-GFP (green) and RFP-Moesin (red), were acquired simultaneously for better time resolution. Lateral views (zstack maximum projections) were taken at 5 min intervals from stage 13 to 15 . Anterior is to the left. Tracheal cell at the base of the stalk gradually over-elongates, forming a long cytoplasmic thread (white arrow). 


\section{Supplementary Figures}

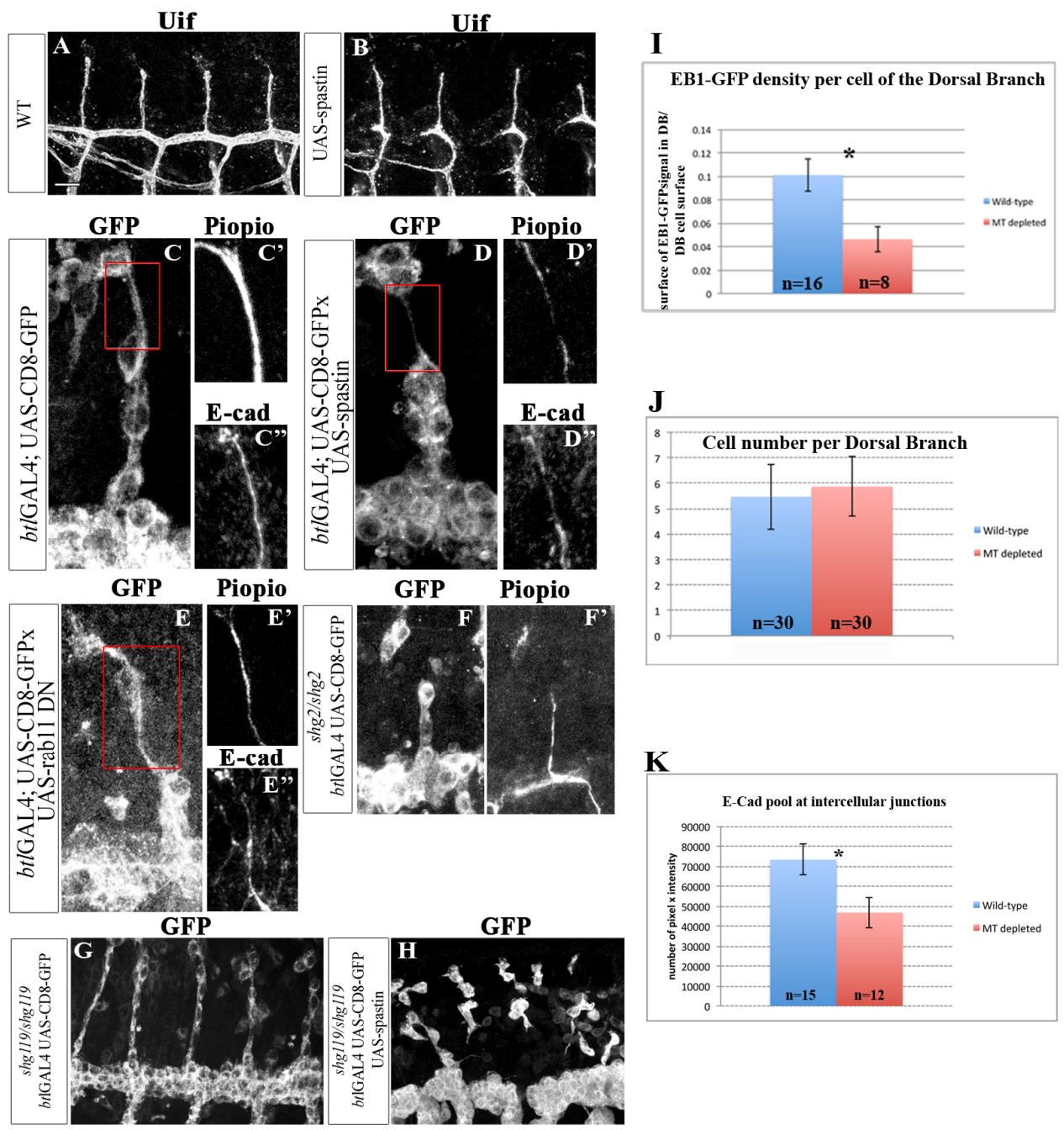

Supplementary Figure S1. MT depletion does not affect lumen deposition but interferes with E-Cad localisation and function

(A, B) Deposition of lumen products, revealed by the anti-Uninflatable (Uif) antibody, is similar in WT (A) and Spastin-overexpressing tracheal cells (B). Expression of the membrane bound form CD8::GFP in WT (C), MT-depleted (D) and Rab11DN overexpression contexts and of the corresponding distribution of the lumen product labelled with Piopio (C', D', E') and with E-Cad (C', D', E'). In the long cytoplasmic thread formed by an over-elongated cell, the lumen and the $\mathrm{AJ}$ are reduced and irregular. (F, F') Expression of the membrane bound form CD8::GFP in mutant embryos for the amorphic allele $s h g^{2}$ (F). The lumen labelled with Piopio (F') is irregular. $(\mathrm{G}, \mathrm{H})$ Tracheal branch outgrowth in homozygous mutant embryos for the hypomorphic allele $\operatorname{shg}^{119}$ alone $(\mathrm{G})$ or associated with Spastin 
overexpression (H). (I) Graph plotting the quantification of the EB1-GFP signal density in WT and MT-depleted tracheal cells of the DB. p-value Test Mann-Whitney $=0.016$. $(\mathrm{J})$ At stage 15, cell numbers in DBs of central metameres A4 to A7 are comparable between WT and MT-depleted embryos (K) Graph showing the quantifications, in WT and MT-depleted contexts, of E-Cad immunofluorescence restricted to intercellular junctions of the DB at stage 16; $\mathrm{p}$-value test Mann-Whitney $=0.019$. 


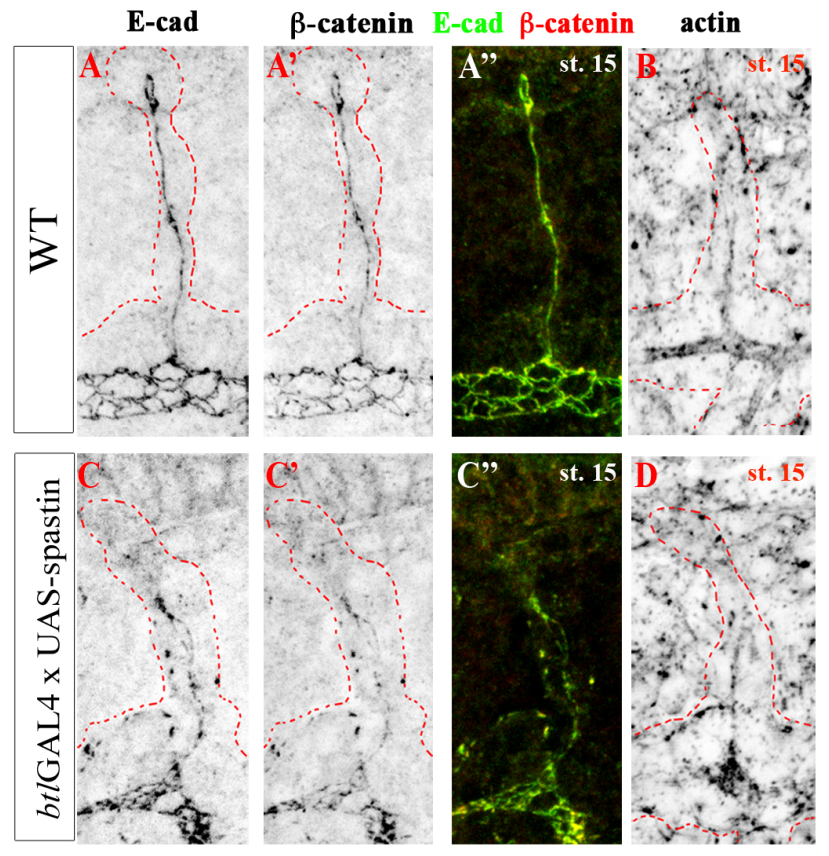

\section{Supplementary Figure S2. Characterisation of E-Cad intracellular accumulation in MT-} depleted tracheal cells

(A-D) DT and DB of stage 15 embryos in WT (A, B) or Spastin overexpression (C, D) contexts. MT depletion induces cytoplasmic accumulation of AJ components as shown using E-Cad and $\beta$-catenin antibodies (compare A', A' to B, B'") but does not significantly alter the apical localisation of actin (compare B to D). E-Cad distribution in WT (E) and Spastin overexpressing (F) embryos at stage 14 is presented. MT depletion produces cytoplasmic accumulation of E-Cad (F). 

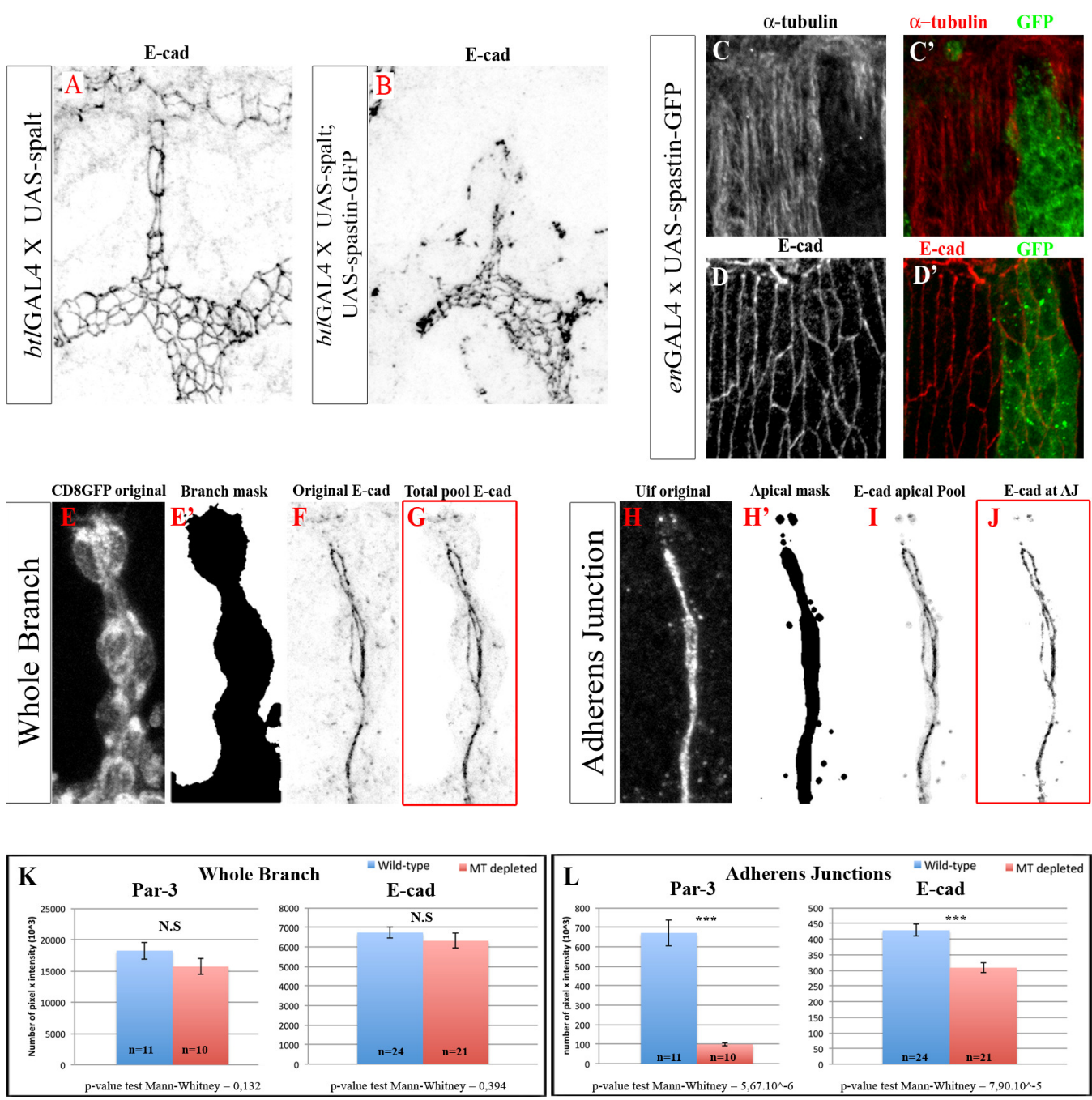

Supplementary Figure S3. MT depletion does not depend on the intercalation state and has no influence on epidermal cells. Details of the macro developed to quantify AJ component levels in tracheal cells

(A) Overexpression of the Spalt transcription factor prevents tracheal cell intercalation as observed by E-Cad staining. Together with Spastin overexpression (B), cell intercalation is still prevented and cytoplasmic accumulation of E-Cad is visible. (C-D') Spastin overexpression in the dorsal part of ectodermal cells using the en-GAL4 driver. As in trachea, strong depolymerisation of MTs is observed (C, C') in stage 15 embryos. However, as opposed to trachea, E-Cad distribution is not altered (D, D'). (E-J) The series of pictures extracted from image processing depict how the macro functions using ImageJ. Z-projections are represented but the macro processes the images section by section. First, the macro is programmed to define the contours of CD8-GFP expression in tracheal cells (E) thus defining 
a branch mask (E'). Within this branch mask, the total pool of E-Cad $(F, G)$ is measured and corresponds to the number of pixels multiplied by their intensity. Second, the macro is programmed to define a mask outlining the luminal staining Uif $(\mathrm{H})$ and corresponding to the apical domain of tracheal cells $\left(\mathrm{H}^{\prime}\right)$. This apical mask is again applied on the E-Cad staining to extract part of the E-Cad corresponding to the apical pool (I). Finally, the apical pool of ECad is determined using as cut-off the mean intensity value of WT AJ staining. This pool of E-Cad at the AJ $(\mathrm{J})$ corresponds to the number of remaining pixels multiplied by their intensity. The same process is used for Par-3 staining and for both in WT and MT-depleted contexts. (K, L) Graphs showing the quantifications of Par-3 and E-Cad immunofluorescence in WT and MT-depleted contexts within the entire DT (K), or restricted to AJs (L). 


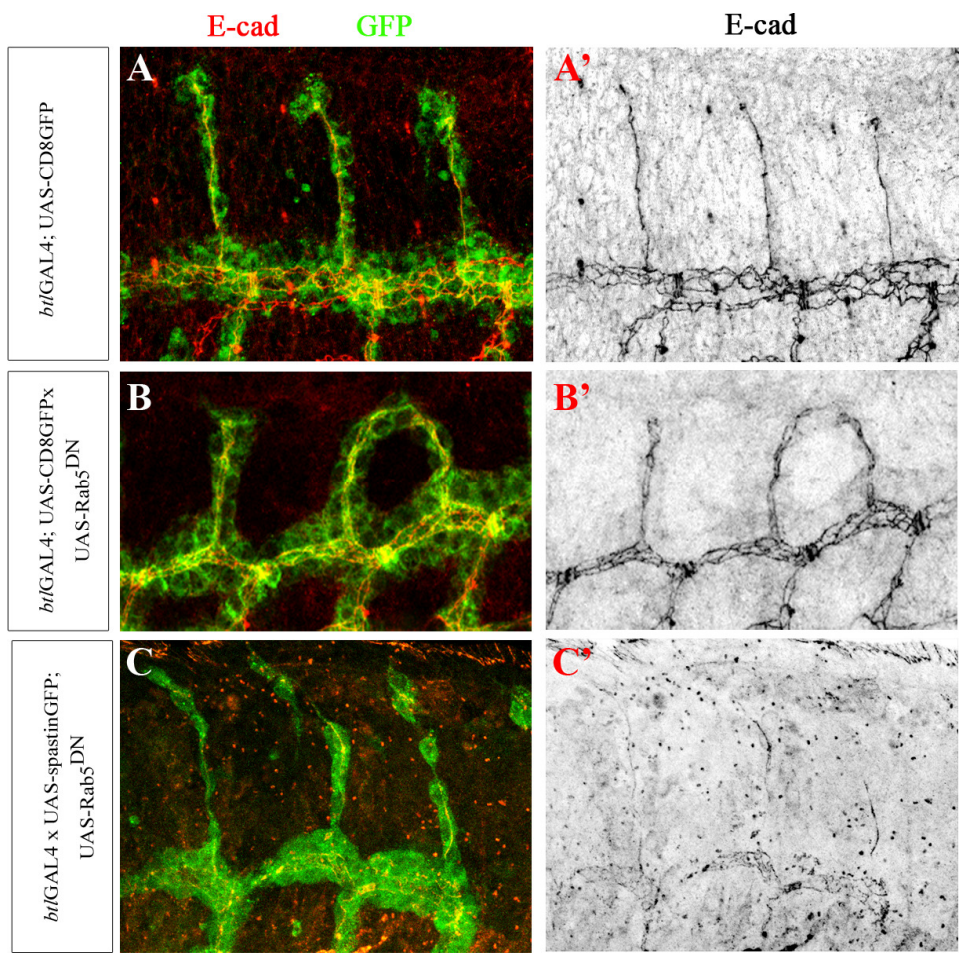

Supplementary Figure S4. Blocking E-Cad internalisation by overexpressing Rab5DN does not prevent trachea cell over-elongation induced by the absence of MTs

Stage 15 embryos expressing the membrane-bound form CD8::GFP (A-C) in tracheal cells and E-Cad localisation (A'-C'). Overexpression of Rab5DN inhibits intercalation in the DB visualized as parallel lines of E-cad (B') compared to a straight line formed by autocellular junctions in controls (A'). Overexpression of Rab5DN in cells depleted of MTs leads to cell over-elongation and branch breaks (C) as in overexpression of Spastin alone. A strong reduction of E-Cad levels at AJs is also observed (C'). 


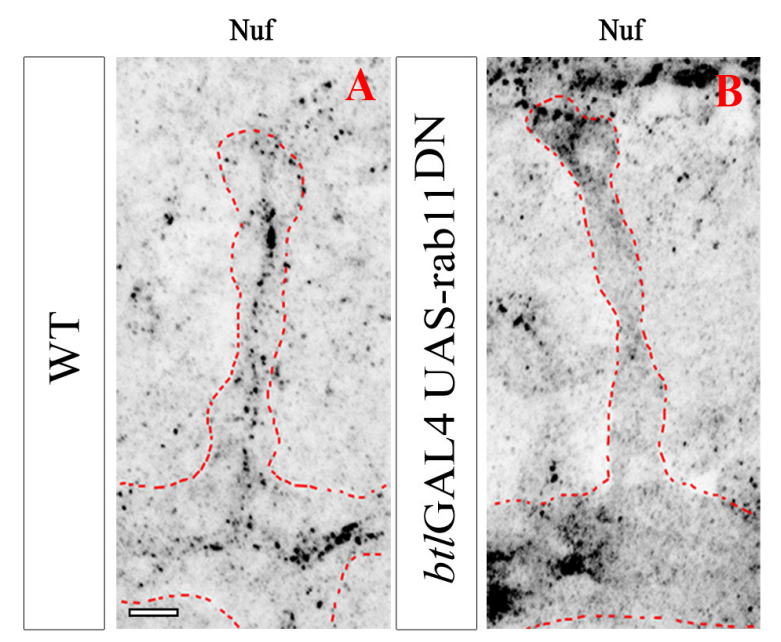

Supplementary Figure S5. Overexpression of Rab11-DN disrupts apical recycling endosome distribution in tracheal cells

Nuf-positive recycling endosomes accumulate apically in the DT and DB at stage 15 of WT embryos (A). Overexpression of Rab11-DN in tracheal cells disrupts recycling endosome distribution (B). 


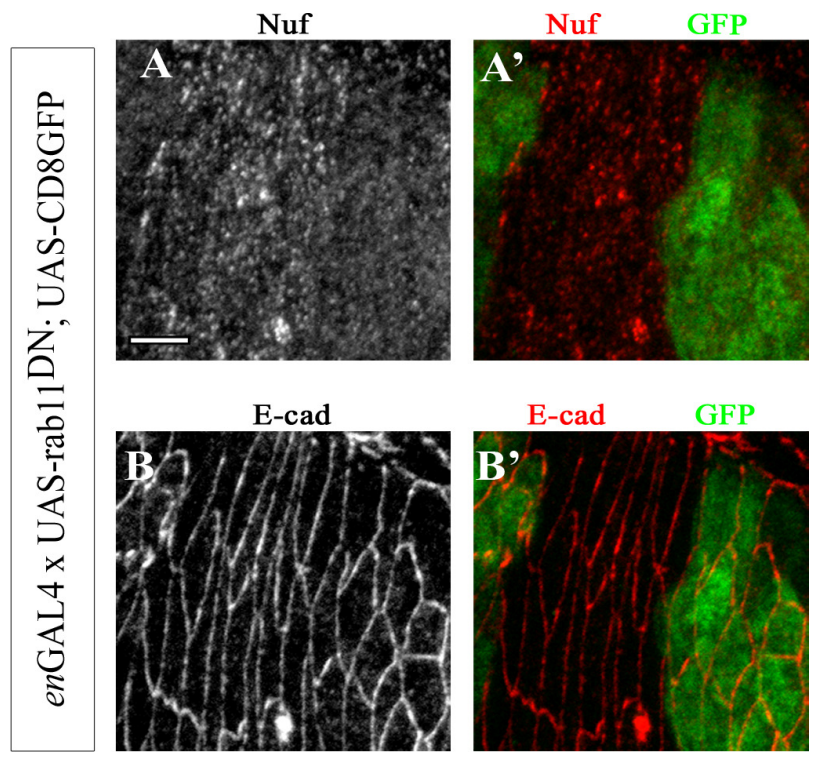

Supplementary Figure S6. Overexpression of Rab11-DN in ectodermal cells affects the distribution of recycling endosomes but not of E-Cad.

Overexpression of Rab11-DN driven by en-GAL4 in ectodermal cells of the dorsal side of stage 15 embryos. As in tracheal cells, spreading of Nuf-positive recycling endosomes is observed (A-A'), but as opposed to tracheal cells, E-Cad distribution is not altered (B-B'). 


\section{Supplementary References}

Brand, A. H. and Perrimon, N. (1993) 'Targeted gene expression as a means of altering cell fates and generating dominant phenotypes', Development 118(2): 401-15.

Brodu, V., Baffet, A. D., Le Droguen, P.-M., Casanova, J. and Guichet, A. (2010) 'A developmentally regulated two-step process generates a noncentrosomal microtubule network in Drosophila tracheal cells', Dev Cell 18(5): 790-801.

Brodu, V., Elstob, P. R. and Gould, A. P. (2004) 'EGF receptor signaling regulates pulses of cell delamination from the Drosophila ectoderm', Dev Cell 7(6): 885-95.

Campos-Ortega, A. J. and Hartenstein, V. (1985) The embryonic development of Drosophila melanogaster: Springer-Verlag, New York.

Cavey, M., Rauzi, M., Lenne, P.-F. and Lecuit, T. (2008) 'A two-tiered mechanism for stabilization and immobilization of E-cadherin', Nature 453(7196): 751-6.

Huang, J., Zhou, W., Dong, W., Watson, A. M. and Hong, Y. (2009) 'From the Cover: Directed, efficient, and versatile modifications of the Drosophila genome by genomic engineering', Proc Natl Acad Sci USA 106(20): 8284-9.

Jankovics, F. and Brunner, D. (2006) 'Transiently reorganized microtubules are essential for zippering during dorsal closure in Drosophila melanogaster', Dev Cell 11(3): 375-85.

Miller, K. G., Field, C. M. and Alberts, B. M. (1989) 'Actin-binding proteins from Drosophila embryos: a complex network of interacting proteins detected by F-actin affinity chromatography', J Cell Biol 109(6 Pt 1): 2963-75.

Ribeiro, C., Neumann, M. and Affolter, M. (2004) 'Genetic control of cell intercalation during tracheal morphogenesis in Drosophila', Current biology : CB 14(24): 2197-207.

Shiga, Y., Tanaka-Matakatsu, M. and Hayashi, S. (1996) 'A nuclear GFP/ $\beta$-galactosidase fusion protein as a marker for morphogenesis in living Drosophila', Develop. Growth Differ. 38: 99-106.

Trotta, N., Orso, G., Rossetto, M. G., Daga, A. and Broadie, K. (2004) 'The hereditary spastic paraplegia gene, spastin, regulates microtubule stability to modulate synaptic structure and function', Current biology : CB 14(13): 1135-47. 